\title{
On the occupation times in a delayed Sparre Andersen risk model with exponential claims
}

\author{
Can Jin ${ }^{1}$, Shuanming $\mathrm{Li}^{2}$ and Xueyuan $\mathrm{Wu}^{2 *}$ \\ ${ }^{1}$ Bond Business School, Bond University, QLD 4229, Australia \\ ${ }^{2}$ Centre for Actuarial Studies, the University of Melbourne, VIC 3010, Australia
}

\begin{abstract}
In this paper, we study the joint Laplace transform of the occupation times in disjoint intervals until ruin in a delayed Sparre Andersen risk model with general inter-claim times and exponential claims. We extend the transformation method in the literature and apply the theoretical fluctuation techniques to derive an explicit expression of the joint Laplace transform under consideration. Further, with the presence of a constant dividend barrier, we derive explicit expressions for the Laplace transforms of the time of ruin and the non-dividend paying duration, namely the total length of non-dividend paying periods prior to ruin. This quantity is of practical interest but has not been studied in the literature to date. Within this paper, all of the Laplace transforms are expressed in terms of scale functions associated with the given spectrally negative Lévy process. Numerical examples are also provided at the end of this paper regarding the Laplace transform of the non-dividend paying duration to illustrate how the distribution of this occupation time behaves in response to varying parameters and the impact of delay on the occupation times comparing with an ordinary Sparre Andersen risk model.
\end{abstract}

Keywords: Sparre Andersen risk model; spectrally negative Lévy process; transformation method; scale function; Laplace transform; constant dividend barrier; dividend paying duration; non-dividend paying duration

(C)2016. This manuscript version is made available under the CC-BY-NC-ND 4.0 license http://creativecommons.org/licenses/by-nc-nd/4.0/

\footnotetext{
*Corresponding author. Email: xueyuanw@unimelb.edu.au; Tel.: 61-3-83447939
} 


\section{Introduction}

In this paper we consider a continuous-time delayed Sparre Andersen risk model with a surplus process defined as

$$
U(t)=u+c t-\sum_{i=1}^{N_{t}} X_{i}, \quad t \geq 0,
$$

where $u \geq 0$ is the initial surplus, $c$ is the premium rate and $\left\{X_{i}\right\}_{i=0}^{\infty}$ is a sequence of independent and identically distributed (i.i.d.) positive random variables (r.v.) denoting the individual claim amounts, with a common distribution function (d.f.) $F$ and probability density function (p.d.f.) $f$. Also, $\left\{N_{t}: t \geq 0\right\}$, which denotes the number of claims up to time $t$, is a delayed renewal process generated by a sequence of inter-claim times $\left\{K_{i}\right\}_{i=1}^{\infty}$, i.e.

$$
N_{t}=\inf \left\{i \geq 1: K_{1}+K_{2}+\cdots+K_{i} \geq t\right\} .
$$

Note here the first claim occurrence time $K_{1}$ is assumed to follow p.d.f. $h_{0}$. And $\left\{K_{i}\right\}_{i=2}^{\infty}$ are assumed to have a common p.f. $H$ (with survival function $\bar{H}$ ), p.d.f. $h$ and Laplace transform (L.T.) $\hat{h}$. We further assume that the two random variable sequences $\left\{K_{i}\right\}_{i=1}^{\infty}$ and $\left\{X_{i}\right\}_{i=1}^{\infty}$ are independent from each other and the positive safety loading condition is satisfied, i.e. $\mathbb{E}\left[K_{i}\right]=(1+\alpha) \mathbb{E}\left[X_{i}\right], \alpha>0, i \in \mathbb{N}^{+} /\{1\}$. One of the central research focuses in risk theory is the studies regarding the time of ruin, i.e. the first time that the surplus level of one insurance company, modeled by the above surplus process $\{U(t): t \geq 0\}$, falls below zero. Here we define the time of ruin as

$$
T_{u}=\inf \{t \geq 0: U(t)<0 \mid U(0)=u\}, \quad u \geq 0,
$$

and the ultimate probability of ruin is defined as $\psi(u)=\mathbb{P}\left(T_{u}<\infty\right)$. A sample path of $\{U(t): t \geq 0\}$ is given in Figure 1 .

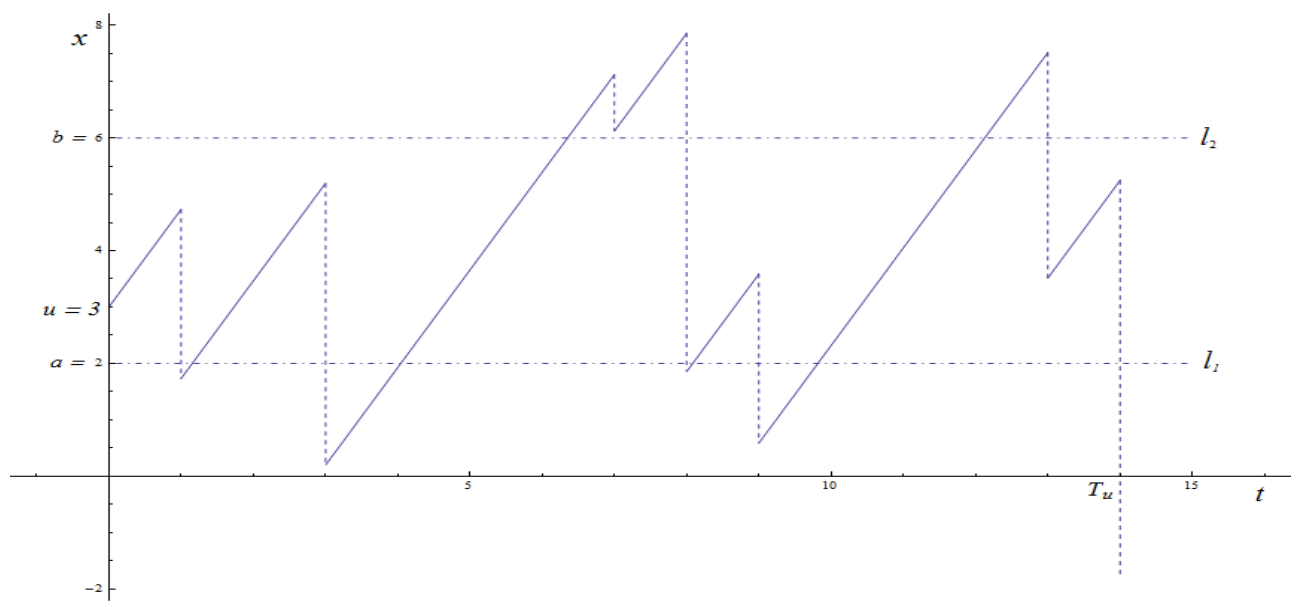

Figure 1: A sample path of $\{U(t): t \geq 0\}$ 
Another research topic that attracts a lot of attention is the well-known expected discounted penalty function (also known as the Gerber-Shiu function) of the given risk process which is defined as

$$
\phi_{\delta}(u)=\mathbb{E}\left[e^{-\delta T_{u}} \omega\left(U\left(T_{u}-\right),\left|U\left(T_{u}\right)\right|\right) \mathbf{1}_{\left\{T_{u}<\infty\right\}}\right], \quad u \geq 0,
$$

where $\delta$ is a force of interest or a Laplace transform argument, and $\mathbf{1}_{\{\cdot\}}$ is an indicator function. Within (1.2), the quantity $\omega\left(U\left(T_{u}-\right),\left|U\left(T_{u}\right)\right|\right)$ can be interpreted as a penalty function at ruin with surplus prior to ruin $U\left(T_{u}-\right)$ and deficit at ruin $\left|U\left(T_{u}\right)\right|$. In particular, when $\omega(x, y)=1,(1.2)$ reduces to the Laplace transform of $T_{u}$ with respect to (w.r.t.) $\delta$,

$$
m_{\delta}(u)=\mathbb{E}\left[e^{-\delta T_{u}} \mathbf{1}_{\left\{T_{u}<\infty\right\}}\right], \quad u \geq 0 .
$$

Let $A$ denote a range of surplus levels. Then

$$
D_{A}:=\int_{0}^{T_{u}} \mathbf{1}_{\{U(t) \in A\}} d t
$$

is the occupation time of $U(t)$ in $A$ before ruin. It records the total amount of time that the surplus level of the insurance company falls within range $A$ before ruin occurs. Consider an arbitrary interval of surplus, $(a, b)$, with $0 \leq a \leq b$. Making use of $D_{A}$, the time of ruin $T_{u}$ can then be decomposed into three occupation times (as shown in Figure 1), i.e.

$$
\begin{aligned}
T_{u} & =\int_{0}^{T_{u}} \mathbf{1}_{\{0<U(t)<a\}} d t+\int_{0}^{T_{u}} \mathbf{1}_{\{a<U(t)<b\}} d t+\int_{0}^{T_{u}} \mathbf{1}_{\{b<U(t)<\infty\}} d t \\
& =D_{(0, a)}+D_{(a, b)}+D_{(b, \infty)} .
\end{aligned}
$$

In particular, when $a=0, D_{(0, b)}$ represents the total duration of surplus level below $b$ before ruin occurs, and correspondingly, $D_{(b, \infty)}$ denotes the duration of surplus above $b$. Therefore, $m_{\delta}(u)$ can be extended to

$$
m_{\delta_{1}, \delta_{2}, \delta_{3}}(u, a, b)=\mathbb{E}\left[e^{-\delta_{1} D_{(0, a)}-\delta_{2} D_{(a, b)}-\delta_{3} D_{(b, \infty)}} \mathbf{1}_{\left\{T_{u}<\infty\right\}}\right],
$$

which is effectively the joint Laplace transform of $D_{(0, a)}, D_{(a, b)}$ and $D_{(b, \infty)}$ with arguments $\delta_{1}, \delta_{2}$ and $\delta_{3}$. Some special cases include

$$
m_{\delta_{1}, \delta_{2}, \delta_{3}}(u, a, b)= \begin{cases}m_{\delta}(u) & \text { if } \delta_{1}=\delta_{2}=\delta_{3}=\delta, \\ m_{1}(u, a) & \text { if } \delta_{2}=\delta_{3}=0, \\ m_{2}(u, a, b) & \text { if } \delta_{1}=\delta_{3}=0, \\ m_{3}(u, b) & \text { if } \delta_{1}=\delta_{2}=0 .\end{cases}
$$

In recent actuarial literature, there has been significant amount of research on the occupation time of an insurer's surplus for some interval $(a, b), a<b$. In risk theory, occupation times are utilized as an enhanced risk management tool to manage insurable risks. As the author pointed out in [14], the recovery time after ruin can assist insurers to determine if it is worth continuing the business. As a generalization of the recovery time, 
the occupation time of the surplus process being in interval $(a, b)$ prior to ruin can be used as an alternative management tool to examine the performance of an insurance portfolio. On one hand, the occupation time in $(0, a)$ (for example, the minimum capital requirement set by regulatory authorities) measures the time an insurer's surplus remaining at critically low levels, which may help to measure the solvency risk; on the other hand, the occupation time in $(b, \infty)$ (for an adequately chosen $b>0$ ) measures the time the company performs well which may help to determine appropriate dividend strategies.

The distribution of negative surplus and the recovery time have been studied in [12] and [11] in the context of the classical risk model. In the spectrally negative Lévy model, [21] and [30] adopted two different approximation schemes to study the Laplace transforms on occupation times. In [21] the authors approximated the occupation time by a union of disjoint random time periods whose Laplace transform can be obtained using solutions to some exit problems while in [30] the spectrally negative Lévy process (SNLP), of which sample paths have unbounded variation, is approximated by a SNLP with sample paths of bounded variation whose Laplace transform for the occupation time can be found directly. [22] generalized the studies on occupation times into Markovian arrival process (MAP) risk models by relaxing the assumption of stationary and independent process increments and obtained a closed-form expression for the Laplace transform of the occupation times. [27] obtained the joint Laplace transforms of the SNLP occupation times over disjoint intervals $(0, a)$ and $(a, b)$ conditional on the two-sided exit stopping times, i.e. either from level 0 or level $b$. The authors adopted a different approach to those used in [21] and [30] in the sense that it associates the Laplace transform of occupation time with a problem concerning the fluctuation of the SNLP observed at two independent sequences of Poisson arrival times. Another major contribution of [30] and [27] is to express the Laplace transforms in terms of scale functions for SNLP. Some other research regarding occupation time include [4], [9], [23], etc. However, as far as we know, the occupation times have not yet been studied in renewal risk models (Sparre Andersen risk models) in which the inter-claim times are i.i.d. and follow a general distribution.

Another main topic in actuarial literature is the risk model involving a barrier dividend strategy. A binomial model with a constant dividend barrier was proposed by [10] and further research on the constant dividend barrier strategies includes [29], [35], [24], [16], [20], [33] and the references therein. [1] studied the distribution of dividend payments till ruin under a Sparre Andersen risk model with generalized Erlang $(n)$ inter-claim times and a constant dividend barrier and derived an integro-differential equation for the moment generating function of the sum of discounted dividend payments before ruin. [26] extended [1] into a Sparre Andersen model with phase-type inter-claim times and showed that the distribution of the total dividend payments before ruin is a mixture of a degenerate distribution at zero and a phase-type distribution. Other types of barrier strategies include threshold strategy in [28], [2] and [7] etc; linear barrier strategy in [13] and [3] etc; nonlinear barrier strategy in [5] and [6] etc. Given the above work, we have only seen studies on the distribution of the dividend paying duration (equivalently the distribution of total dividends) with the presence of a constant dividend barrier, the corresponding non-dividend paying duration has never been studied. 
In [8], the authors derived a closed-form expression for the distribution of the time to ruin for a delayed Sparre Andersen risk model with exponentially distributed claims by transforming the original boundary crossing problem to an equivalent one on linear lower boundary crossing by a spectrally positive Lévy process. By extending [8] to Erlang distributed claims, [34] obtained a formula for the joint density of the time to ruin and the number of claims until ruin. In this paper, we adopt and extend the transformation method to transform a Sparre Andersen risk process with exponential claims into a Cramér Lundberg process (a special case of SNLP) with arbitrarily distributed claims. After establishing the relationships among the occupation times in the original and transformed models, we obtain the Laplace transforms of the occupation times in the original risk model based on the ones in the transformed Cramér Lundberg model. A similar transform is done to a delayed Sparre Andersen risk process with a constant dividend barrier so that the Laplace transforms of the time of ruin and the non-dividend paying duration are derived.

The rest of the paper is organized as follows. In Section 2 we briefly review the spectrally negative Lévy process, the scale function and some relevant fluctuation identities. Then we perform an equivalent transformation and establish the relationships among the occupation times in the original and transformed models in Section 3. By working with the transformed Cramér Lundberg model, we derive the joint Laplace transform of the occupation times in three disjoint intervals prior to ruin in Section 4. In Section 5, a Sparre Andersen risk model with exponential claims in the presence of a constant dividend barrier is considered and Laplace transforms of the time of ruin and the non-dividend paying duration are derived respectively. Lastly in Section 6, we present some numerical calculations on the Laplace transform of the non-dividend paying duration to illustrate how the distribution of this occupation time behaves in response to varying parameters and the impact of delay on the occupation times comparing with an ordinary Sparre Andersen risk model.

\section{Fluctuation identities of spectrally negative Lévy processes and associated scale functions}

In this section we briefly review the spectrally negative Lévy processes and the associated scale functions. Let $\{V(t): t \geq 0\}$ on the filtered probability space $\left(\Omega,\left\{\mathcal{F}_{t}\right\}_{t \geq 0}, \mathbb{P}\right)$ denote a spectrally negative Lévy process, which is a process with stationary and independent increments and no positive jumps. In this paper, we exclude the case of being the negative of a subordinator, i.e. $\{V(t): t \geq 0\}$ cannot have decreasing paths. We denote the law of $\{V(t): t \geq 0\}$ with $V(0)=\nu$ as $\mathbb{P}_{\nu}$ and the corresponding expectation as $\mathbb{E}_{\nu}$. For $\nu=0$, we write $\mathbb{P}$ and $\mathbb{E}$ for simplicity. Based on our assumption, the Laplace exponent $\Psi$ of the Lévy process exists and is defined through

$$
\mathbb{E}\left[e^{s V(t)}\right]=e^{t \Psi(s)}, \quad s, t \geq 0,
$$

and satisfies

$$
\Psi(s)=\gamma s+\frac{1}{2} \sigma^{2} s^{2}+\int_{0}^{\infty}\left(e^{-s z}-1+s z \mathbf{1}_{\{0<z \leq 1\}}\right) \Pi(d z), \quad \gamma \in \mathbb{R}, \sigma \geq 0,
$$


and $\Pi$ is a $\sigma$-finite measure of $(0, \infty)$ such that

$$
\int_{0}^{\infty}\left(1 \wedge z^{2}\right) \Pi(d z)<\infty
$$

where $x \wedge y$ denotes the minimum of $x$ and $y$. And $\Pi$ is known as the Lévy measure of $V(t)$, while $(\gamma, \sigma, \Pi)$ is referred to as the Lévy triplet of $\{V(t): t \geq 0\}$. Throughout this paper we define the Lévy measure in such a way that it is a measure on the positive half real line instead of the negative half real line. The process $\{V(t): t \geq 0\}$ has paths of bounded variation if and only if $\sigma=0$ and $\int_{0}^{1} z \Pi(d z)<\infty$. One example is the Cramér-Lundberg process, i.e.

$$
V(t)=c t-\sum_{i=1}^{M_{t}} X_{i}, \quad t \geq 0
$$

where $\left\{M_{t}: t \geq 0\right\}$ is a Poisson process with parameter $\lambda . V(t)$ has the Laplace exponent

$$
\Psi(s)=c s-\lambda \int_{0}^{\infty}\left(1-e^{-s x}\right) f(x) d x,
$$

and the safety loading condition on $V(t)$ can be expressed in terms of $\Psi$ as

$$
\frac{d}{d s} \Psi(0+)=\lim _{s \rightarrow 0+} \frac{\Psi(s)}{s}=c-\lambda \mathbb{E}\left[X_{1}\right]>0 .
$$

$\Psi$ is strictly convex and $\lim _{y \rightarrow \infty} \Psi(y)=\infty$, and there exists a function $\rho:[0, \infty) \rightarrow[0, \infty)$ defined by $\rho(\delta)=\sup \{y \geq 0 \mid \Psi(y)=\delta\}$ (the right-inverse) such that

$$
\Psi(\rho(\delta))=\delta, \quad \delta \geq 0,
$$

with $\rho(\delta)=0$ if and only if $\delta=0$ and $\frac{d}{d s} \Psi(0+)=\mathbb{E}[V(1)] \geq 0 . \quad \rho(\delta)$ is known to be the non-negative solution of the Lundberg's fundamental equation for the classical risk model

$$
c s-(\lambda+\delta)+\lambda \hat{f}(s)=0, \quad \delta, s \geq 0 .
$$

More information can be found in [19].

Then we recall the definition of the $\delta$-scale function $W^{(\delta)}(x), x \in[0, \infty)$. For $\delta \geq 0$, the $\delta$-scale function of the process $\{V(t): t \geq 0\}$ is defined as a continuous function on $[0, \infty)$ with a Laplace transform given by

$$
\int_{0}^{\infty} e^{-s x} W^{(\delta)}(x) d x=\frac{1}{\Psi(s)-\delta}, \quad s>\rho(\delta) .
$$

This function is unique, positive, strictly increasing for $x \geq 0$ and is continuous for $\delta \geq 0$. We extend $W^{(\delta)}$ to the whole real line by setting $W^{(\delta)}(x)=0$ for $x<0$ and write $W:=W^{(0)}$ when $\delta=0$. The initial values of $W^{(\delta)}$ are known to be

$$
W^{(\delta)}(0)=\lim _{x \rightarrow 0} W^{(\delta)}(x)= \begin{cases}1 / c, & \sigma=0 \quad \text { and } \quad \int_{0}^{1} z \Pi(d z)<\infty \\ 0, & \text { otherwise }\end{cases}
$$


which corresponds to the Lévy processes with bounded variation and unbounded variation respectively. Note that $\gamma+\int_{0}^{1} z \Pi(d z)=c$, the drift of $\{V(t): t \geq 0\}$. Another function used frequently is

$$
Z^{(\delta)}(x)=1+\delta \int_{0}^{x} W^{(\delta)}(y) d y, \quad x \geq 0
$$

where $Z^{(\delta)}(x):=1$ for $x<0$. Similarly we write $Z:=Z^{(0)}$. Two asymptotic behaviors are known as

$$
\lim _{x \rightarrow \infty} \frac{Z^{(\delta)}(x)}{W^{(\delta)}(x)}=\frac{\delta}{\rho(\delta)} \quad \text { and } \quad \lim _{x \rightarrow \infty} \frac{W^{(\delta)}(x-a)}{W^{(\delta)}(x)}=e^{-\rho(\delta) a} .
$$

To utilize the scale functions, we introduce the following first passage time r.v.s

$$
\tau_{a}^{-}=\inf \{t>0: V(t)<a\}, \quad \tau_{a}^{+}=\inf \{t>0: V(t)>a\},
$$

with the convention $\inf \emptyset=\infty$. It is well known in the existing literature that if $a>0$ and $\nu \leq a$, the solution to the two-sided exit problem is given as

$$
\mathbb{E}_{\nu}\left[e^{-\delta \tau_{a}^{+}} \mathbf{1}_{\left\{\tau_{a}^{+}<\tau_{0}^{-}\right\}}\right]=\frac{W^{(\delta)}(\nu)}{W^{(\delta)}(a)},
$$

and

$$
\mathbb{E}_{\nu}\left[e^{-\delta \tau_{0}^{-}} \mathbf{1}_{\left\{\tau_{0}^{-}<\tau_{a}^{+}\right\}}\right]=Z^{(\delta)}(\nu)-Z^{(\delta)}(a) \frac{W^{(\delta)}(\nu)}{W^{(\delta)}(a)} .
$$

Several auxiliary functions introduced in [30] are used in this paper. One equality holds:

$$
(q-p) \int_{0}^{a} W^{(p)}(a-y) W^{(q)}(y) d y=W^{(q)}(a)-W^{(p)}(a),
$$

then for $p, q \geq 0$ and $x \in \mathbb{R}$, we have

$$
\begin{aligned}
\mathcal{W}_{a}^{(p, q)}(x) & :=W^{(p+q)}(x)-q \int_{0}^{a} W^{(p+q)}(x-y) W^{(p)}(y) d y \\
& =W^{(p)}(x)+q \int_{a}^{x} W^{(p+q)}(x-y) W^{(p)}(y) d y \\
\mathcal{Z}_{a}^{(p, q)}(x) & :=W^{(p+q)}(x)-q \int_{a}^{x} W^{(p+q)}(y) W^{(p)}(x-y) d y \\
& =W^{(p)}(x)+q \int_{0}^{a} W^{(p+q)}(y) W^{(p)}(x-y) d y .
\end{aligned}
$$

For $p \geq 0$ and $q \in \mathbb{R}$ such that $p+q \geq 0$, we define an increasing function

$$
\mathcal{H}^{(p, q)}(x)=e^{\rho(p) x}\left(1+q \int_{0}^{x} e^{-\rho(p) y} W^{(p+q)}(y) d y\right), \quad x \in \mathbb{R},
$$


with $\mathcal{H}^{(p, q)}(x)=e^{\rho(p) x}$ for $x \leq 0$. The Laplace transform of $\mathcal{H}^{(p, q)}$ on $[0, \infty)$ is explicitly given as

$$
\int_{0}^{\infty} e^{-\lambda x} \mathcal{H}^{(p, q)}(x) d x=\frac{1}{\lambda-\rho(p)}\left(1+\frac{q}{\Psi(\lambda)-p-q}\right), \quad \lambda>\rho(p+q) .
$$

Given a positive and measurable function $\beta^{(q)}(x)$ on $\mathbb{R}$ and $q \geq 0$, [30] proved that

$$
\mathbb{E}_{\nu}\left[e^{-q \tau_{a}^{-}} \beta^{(q)}\left(V\left(\tau_{a}^{-}\right)\right) \mathbf{1}_{\left\{\tau_{a}^{-}<\tau_{b}^{+}\right\}}\right]=\beta^{(q)}(\nu)-\frac{W^{(q)}(\nu-a)}{W^{(q)}(b-a)} \beta^{(q)}(b), \quad 0 \leq a \leq \nu \leq b .
$$

Furthermore, if $\{V(t): t \geq 0\}$ has paths of bounded variation, for $p, q \geq 0$,

$$
\begin{aligned}
& \mathbb{E}_{\nu}\left[e^{-p \tau_{a}^{-}} \beta^{(q)}\left(V\left(\tau_{a}^{-}\right)\right) \mathbf{1}_{\left\{\tau_{a}^{-}<\tau_{b}^{+}\right\}}\right] \\
& =\beta^{(q)}(\nu)-(q-p) \int_{a}^{\nu} W^{(p)}(\nu-y) \beta^{(q)}(y) d y \\
& -\frac{W^{(p)}(\nu-a)}{W^{(p)}(b-a)}\left(\beta^{(q)}(b)-(q-p) \int_{a}^{b} W^{(p)}(b-y) \beta^{(q)}(y) d y\right) .
\end{aligned}
$$

Readers may refer to [18] for more details about the spectrally negative Lévy process and [17] for examples and numerical techniques related to the computation of scale functions.

\section{An equivalent transformation}

Within the rest of this paper, we shall assume that the claim size $X_{i}, i \in \mathbb{N}$, follows an exponential distribution with parameter $\lambda$, i.e. $f(x)=\lambda e^{-\lambda x}, x \geq 0$. In this section, we extend the transformation method in [8] and transform our original delayed Sparre Andersen risk process into a Cramér Lundberg process by constructing a new system of coordinates. By doing so, we aim to study the occupation times in the original model via the occupation times in the transformed model.

Assume $K_{1}$ takes value $\xi$ (a constant). Following the transformation method developed in [8], we translate the origin to $(u, \xi)$ and rotate Figure 1 anti-clockwise through 90 degrees by swapping the roles of the time and the space. In mathematical language, we define

$$
\left\{\begin{array}{l}
y=t-\xi \\
s=-x+u+c t
\end{array}\right.
$$

and in this $(s, y)$ system of coordinates, the trajectory of our process $\{U(t): t \geq 0\}$ becomes a step-wise compound Poisson process with positive jumps, i.e.

$$
U(s)=\sum_{i=1}^{N_{s}^{*}} K_{i}, \quad s \geq 0,
$$


where $N_{s}^{*}=\inf \left\{j \geq 1: X_{1}+\cdots+X_{j} \geq s\right\}$ is a Poisson process with parameter $\lambda$ that counts the number of positive jumps by 'time' $s$. Following the transformation, the three lines in the original $(t, x)$ system of coordinates, i.e. $l: x=0, l_{1}: x=a$ and $l_{2}: x=b$, have their trajectories in the $(s, y)$ system of coordinates respectively as follows:

$$
l: y=\frac{s-u-c \xi}{c}, \quad l_{3}: y=\frac{s+a-u-c \xi}{c}, \quad l_{4}: y=\frac{s+b-u-c \xi}{c} .
$$

Therefore the original ruin problem becomes the problem of first crossing time of $l$ by the trajectory $\{U(s): s \geq 0\}$. The new stopping time is defined as

$$
\tau=\inf \left\{s>0: U(s) \leq \frac{s-u-c \xi}{c}\right\},
$$

and since the point $(\tau, \tau-\xi)$ satisfies the equation of $l$, i.e.

$$
T_{u}-\xi=\frac{\tau-u-c \xi}{c}
$$

we have

$$
T_{u}=\frac{\tau-u}{c} .
$$

Now, we 'flip around' the $(s, y)$ system of coordinates and construct a new one:

$$
\left\{\begin{array}{l}
z=u^{\prime}+s-c y \\
s=s
\end{array}\right.
$$

where $u^{\prime}$ denotes the new 'initial surplus' defined as

$$
u^{\prime}=b+a-u-c \xi
$$

In this new system of coordinates, the trajectory of $\{U(t): t \geq 0\}$ becomes

$$
U^{\prime}(s)=u^{\prime}+s-c \sum_{i=1}^{N_{s}^{*}} K_{i}, \quad s \geq 0,
$$

and the lines $l, l_{3}$ and $l_{4}$ are transformed into

$$
l^{*}: z=a+b, \quad l_{5}: z=b, \quad l_{6}: z=a .
$$

The first hitting time $\tau$ is re-defined in this new $(s, z)$ system of coordinates as

$$
\tau=\inf \left\{s>0: U^{\prime}(s)>a+b\right\}
$$

and a sample path of $\left\{U^{\prime}(s): s \geq 0\right\}$ is given below: 


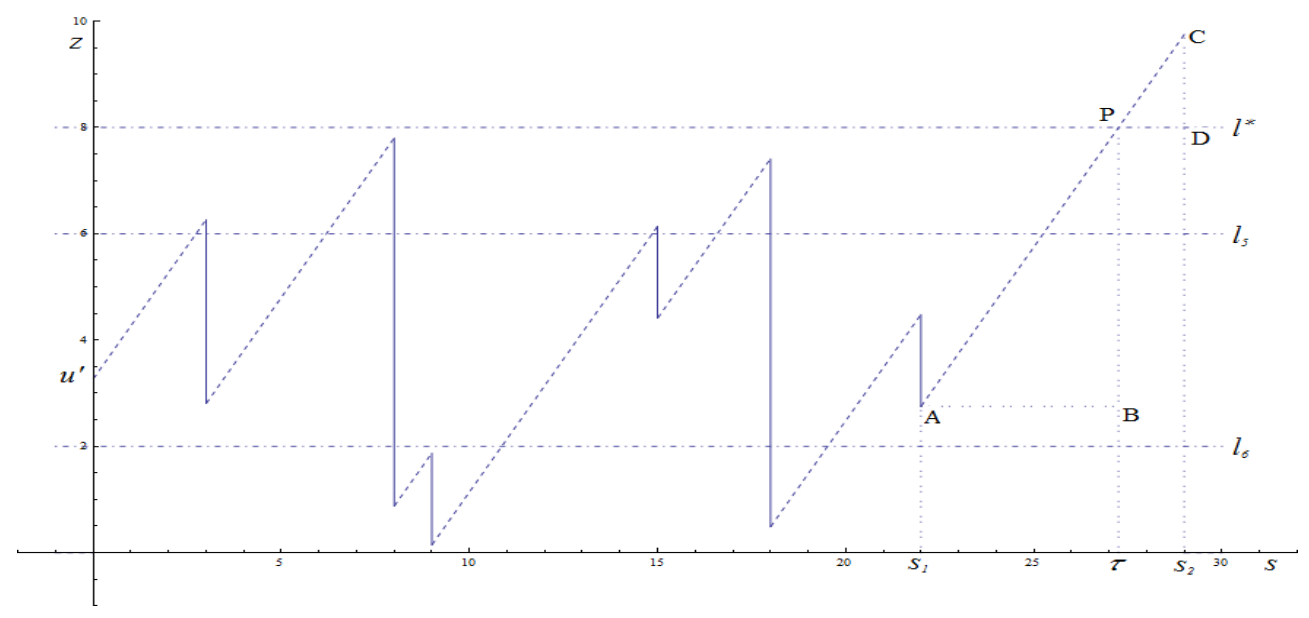

Figure 2: A sample path of $\left\{U^{\prime}(s): s \geq 0\right\}$

As shown in Figure 2, $\left\{U^{\prime}(s): s \geq 0\right\}$ is a Cramér Lundberg process starting with an initial surplus $u^{\prime}$, increases with a constant premium rate 1 and has negative jumps modeled by the Poisson process $N_{s}^{*}$ (number of jumps) and the distribution $H$ (jump sizes). The whole process stops when it up-crosses $l^{*}$, which is equivalent to ruin in the original risk model by construction. Note that the triangles $A P B$ and $C P D$ are isosceles right triangles since the premium rate is 1 .

In the following, we shall present a couple of results generated by the above transformation. Define $\bar{m}_{\delta^{*}}\left(u^{\prime}\right)$ as the Laplace transform of $\tau$ :

$$
\bar{m}_{\delta^{*}}\left(u^{\prime}\right)=\mathbb{E}_{u^{\prime}}\left[e^{-\delta^{*} \tau} \mathbf{1}_{\{\tau<\infty\}}\right],
$$

then we have the following theorem:

Theorem 1 The Laplace transform of time of ruin in the delayed Sparre Andersen risk model (1.1) can be expressed as

$$
m_{\delta}(u)=\int_{0}^{\infty} h_{0}(\xi) e^{\delta^{*} u} \bar{m}_{\delta^{*}}\left(u^{\prime}\right) d \xi, \quad \delta>0, \delta^{*}=\delta / c
$$

Proof. The proof is straight forward by applying (3.3).

Define $D_{A}^{*}$ to be the occupation time of $\left\{U^{\prime}(s): s \geq 0\right\}$ in $A$ before $\left\{U^{\prime}(s): s \geq 0\right\}$ passes the level $a+b$, i.e. $D_{A}^{*}=\int_{0}^{\tau} \mathbf{1}_{\left\{-\infty<U^{\prime}(s) \in A\right\}} d s$. To study $D_{(0, a)}, D_{(a, b)}$ and $D_{(b, \infty)}$ in the original surplus process $U(t)$, we need to consider the intervals $(-\infty, a),(a, b)$ and $(b, a+b)$ regarding process $\left\{U^{\prime}(s): s \geq 0\right\}$. And the joint Laplace transform of $D_{(-\infty, a)}^{*}$, $D_{(a, b)}^{*}$ and $D_{(b, a+b)}^{*}$ has the form

$$
\bar{m}_{\delta_{1}^{*}, \delta_{2}^{*}, \delta_{3}^{*}}\left(u^{\prime}, a, b\right)=\mathbb{E}_{u^{\prime}}\left[e^{-\delta_{1}^{*} D_{(-\infty, a)}^{*}-\delta_{2}^{*} D_{(a, b)}^{*}-\delta_{3}^{*} D_{(b, a+b)}^{*}} \mathbf{1}_{\{\tau<\infty\}}\right],
$$


where $u^{\prime}<a+b$ and $\delta_{1}^{*}, \delta_{2}^{*}, \delta_{3}^{*}>0$. The Laplace transform of these individual durations are

$$
\left\{\begin{array}{l}
\bar{m}_{\delta_{1}^{*}}\left(u^{\prime}, a, b\right)=\mathbb{E}_{u^{\prime}}\left[e^{-\delta_{1}^{*} D_{(-\infty, a)}^{*}} \mathbf{1}_{\{\tau<\infty\}}\right], \\
\bar{m}_{\delta_{2}^{*}}\left(u^{\prime}, a, b\right)=\mathbb{E}_{u^{\prime}}\left[e^{-\delta_{2}^{*} D_{(a, b)}^{*}} \mathbf{1}_{\{\tau<\infty\}}\right], \\
\bar{m}_{\delta_{3}^{*}}\left(u^{\prime}, a, b\right)=\mathbb{E}_{u^{\prime}}\left[e^{-\delta_{3}^{*} D_{(b, a+b)}^{*}} \mathbf{1}_{\{\tau<\infty\}}\right] .
\end{array}\right.
$$

From the equivalent transformation we observe that the occupation times $D_{(0, a)}, D_{(a, b)}$ and $D_{(b, \infty)}$ in the original model (1.1) are scaled by the factor $c$ due to the swap of the coordinates. The initial surplus $u$ is also transformed into 'time' $u$ and hence by conditioning on the relative sizes of $u, a$ and $b$, we establish the relationships between the occupation times in the original model (1.1) and the transformed one (3.6).

Corollary 1 When $0<u<a<b<\infty,\left\{U^{\prime}(s): s \geq 0\right\}$ spends the whole 'time' $u$ in the interval $(0, a)$, so that

$$
\left\{\begin{array}{l}
D_{(-\infty, a)}^{*}=c D_{(b, \infty)} \\
D_{(a, b)}^{*}=c D_{(a, b)} \\
D_{(b, a+b)}^{*}=c D_{(0, a)}+u
\end{array}\right.
$$

When $0<a<u<b<\infty,\left\{U^{\prime}(s): s \geq 0\right\}$ spends 'time' $u$ in two intervals, i.e. $(0, a)$ and $(a, b)$, then we have

$$
\left\{\begin{array}{l}
D_{(-\infty, a)}^{*}=c D_{(b, \infty)}, \\
D_{(a, b)}^{*}=c D_{(a, b)}+(u-a), \\
D_{(b, a+b)}^{*}=c D_{(0, a)}+a .
\end{array}\right.
$$

When $0<a<b<u<\infty,\left\{U^{\prime}(s): s \geq 0\right\}$ spends 'time' $u$ in all the three intervals, therefore we need to adjust all the three occupation times

$$
\left\{\begin{array}{l}
D_{(-\infty, a)}^{*}=c D_{(b, \infty)}+(u-b) \\
D_{(a, b)}^{*}=c D_{(a, b)}+(b-a) \\
D_{(b, a+b)}^{*}=c D_{(0, a)}+a .
\end{array}\right.
$$

As a result of Corollary 1 and Theorem 1, we have the following result.

Theorem 2 The joint Laplace transform of occupation times, $m_{\delta_{1}, \delta_{2}, \delta_{3}}(u, a, b)$, in the delayed Sparre Andersen risk model (1.1) can be expressed as

$$
m_{\delta_{1}, \delta_{2}, \delta_{3}}(u, a, b)=\int_{0}^{\infty} h_{0}(\xi) B(u, a, b) \bar{m}_{\delta_{1}^{*}, \delta_{2}^{*}, \delta_{3}^{*}}\left(u^{\prime}, a, b\right) d \xi
$$

where $B(u, a, b)$ is defined as

$$
B(u, a, b)= \begin{cases}e^{\delta_{1}^{*} u}, & 0<u<a<b<\infty, \\ e^{\delta_{1}^{*} a+\delta_{2}^{*}(u-a)}, & 0<a<u<b<\infty, \\ e^{\delta_{1}^{*} a+\delta_{2}^{*}(b-a)+\delta_{3}^{*}(u-b)}, & 0<a<b<u<\infty .\end{cases}
$$




\section{Remarks:}

- By setting $\delta_{1}, \delta_{2}$ or $\delta_{3}$ to 0 (or equivalently, $\delta_{1}^{*}, \delta_{2}^{*}$ or $\delta_{3}^{*}$ to 0 ), we can obtain the corresponding (joint) Laplace transforms of $D_{(0, a)}, D_{(a, b)}$ or $D_{(b, \infty)}$ respectively.

- Alternatively, one can perform the transformation via the fluid flow approach used in [31] or [32], through which the relationship (3.4) can be obtained as well. However, we claim that adopting the equivalent transformation method makes it easier to find the relationships among the occupation time random variables in the sense that through the transformation, the boundaries of the intervals are translated automatically so we obtain the new boundaries of the corresponding intervals immediately after the transformation.

In the following section, we shall derive the explicit expression for $\bar{m}_{\delta_{1}^{*}, \delta_{2}^{*}, \delta_{3}^{*}}\left(u^{\prime}, a, b\right)$.

\section{Joint Laplace transform of occupation times in the trans- formed Cramér-Lundberg risk model}

In this section, we shall derive an explicit expression for $\bar{m}_{\delta_{1}^{*}, \delta_{2}^{*}, \delta_{3}^{*}}\left(u^{\prime}, a, b\right)$ in terms of scale functions by utilizing the properties of the Lévy process described in Section 2 .

Theorem 3 The joint Laplace transform of $D_{(-\infty, a)}^{*}, D_{(a, b)}^{*}$ and $D_{(b, a+b)}^{*}$ for the process $\left\{U^{\prime}(s): s \geq 0\right\}$, has an explicit expression, for $\delta_{1}^{*}, \delta_{2}^{*}, \delta_{3}^{*} \geq 0$,

$$
\bar{m}_{\delta_{1}^{*}, \delta_{2}^{*}, \delta_{3}^{*}}\left(u^{\prime}, a, b\right)=\frac{\mathcal{H}^{\left(\delta_{1}^{*}, \delta_{2}^{*}-\delta_{1}^{*}\right)}\left(u^{\prime}-a\right)-\left(\delta_{2}^{*}-\delta_{3}^{*}\right) \int_{b}^{u^{\prime}} W^{\left(\delta_{3}^{*}\right)}\left(u^{\prime}-z\right) \mathcal{H}^{\left(\delta_{1}^{*}, \delta_{2}^{*}-\delta_{1}^{*}\right)}(z-a) d z}{\mathcal{H}^{\left(\delta_{1}^{*}, \delta_{2}^{*}-\delta_{1}^{*}\right)}(b)-\left(\delta_{2}^{*}-\delta_{3}^{*}\right) \int_{b}^{a+b} W^{\left(\delta_{3}^{*}\right)}(a+b-z) \mathcal{H}^{\left(\delta_{1}^{*}, \delta_{2}^{*}-\delta_{1}^{*}\right)}(z-a) d z} .
$$

Proof. We begin the proof by considering the Cramér-Lundberg process $\{V(t): t \geq 0\}$ described in Section 2. Here $\{V(t): t \geq 0\}$ takes the form of $\left\{U^{\prime}(s): s \geq 0\right\}$, so the corresponding results on $\{V(t): t \geq 0\}$ can be applied to $\left\{U^{\prime}(s): s \geq 0\right\}$ directly. The first passage times of $\left\{U^{\prime}(s): s \geq 0\right\}$ are defined in the same way as in (2.2). It is worth noting that the first hitting time $\tau$ is the same as $\tau_{a+b}^{+}$according to (2.2) but we will keep using $\tau$ thereafter for simplicity.

For $0 \leq a<b$ and $q_{1}, q_{2}, q_{3} \geq 0$, we define

$$
w\left(u^{\prime}\right)=\mathbb{E}_{u^{\prime}}\left[e^{-q_{1} D_{(0, a)}^{*}-q_{2} D_{(a, b)}^{*}-q_{3} D_{(b, a+b)}^{*}} \mathbf{1}_{\left\{\tau<\tau_{0}^{-}\right\}}\right] .
$$

(1) For $0 \leq u^{\prime}<a$, by the strong Markov property of $\left\{U^{\prime}(s): s \geq 0\right\}$ and the fact that $\left\{U^{\prime}(s): s \geq 0\right\}$ is skip-free upward, we can get

$$
w\left(u^{\prime}\right)=\mathbb{E}_{u^{\prime}}\left[e^{-q_{1} \tau_{a}^{+}} \mathbf{1}_{\left\{\tau_{a}^{+}<\tau_{0}^{-}\right\}}\right] w(a)=w(a) \frac{W^{\left(q_{1}\right)}\left(u^{\prime}\right)}{W^{\left(q_{1}\right)}(a)} .
$$

Note that (4.1) also holds for $u^{\prime}<0$. 
(2) Similarly, for $a \leq u^{\prime}<b$, using (4.1)

$$
\begin{aligned}
w\left(u^{\prime}\right)= & w(b) \mathbb{E}_{u^{\prime}}\left[e^{-q_{2} \tau_{b}^{+}} \mathbf{1}_{\left\{\tau_{b}^{+}<\tau_{a}^{-}\right\}}\right]+\mathbb{E}_{u^{\prime}}\left[e^{-q_{2} \tau_{a}^{-}} w\left(V\left(\tau_{a}^{-}\right)\right) \mathbf{1}_{\left\{\tau_{a}^{-}<\tau_{b}^{+}\right\}}\right] \\
= & \frac{w(a)}{W^{\left(q_{1}\right)}(a)} \mathbb{E}_{u^{\prime}}\left[e^{-q_{2} \tau_{a}^{-}} W^{\left(q_{1}\right)}\left(V\left(\tau_{a}^{-}\right)\right) \mathbf{1}_{\left\{\tau_{a}^{-}<\tau_{b}^{+}\right\}}\right] \\
& +w(b) \frac{W^{\left(q_{2}\right)}\left(u^{\prime}-a\right)}{W^{\left(q_{2}\right)}(b-a)}
\end{aligned}
$$

According to (2.3), (2.4) and (2.7), we know that

$$
\begin{aligned}
& \mathbb{E}_{u^{\prime}}\left[e^{-q_{2} \tau_{a}^{-}} W^{\left(q_{1}\right)}\left(V\left(\tau_{a}^{-}\right)\right) \mathbf{1}_{\left\{\tau_{a}^{-}<\tau_{b}^{+}\right\}}\right] \\
& =W^{\left(q_{1}\right)}\left(u^{\prime}\right)-\left(q_{1}-q_{2}\right) \int_{a}^{u^{\prime}} W^{\left(q_{2}\right)}\left(u^{\prime}-y\right) W^{\left(q_{1}\right)}(y) d y \\
& -\frac{W^{\left(q_{2}\right)}\left(u^{\prime}-a\right)}{W^{\left(q_{2}\right)}(b-a)}\left[W^{\left(q_{1}\right)}(b)-\left(q_{1}-q_{2}\right) \int_{a}^{b} W^{\left(q_{2}\right)}(b-y) W^{\left(q_{1}\right)}(y) d y\right] \\
& = \begin{cases}\mathcal{W}_{a}^{\left(q_{1}, q_{2}-q_{1}\right)}\left(u^{\prime}\right)-\frac{W^{\left(q_{2}\right)}\left(u^{\prime}-a\right)}{W^{\left(q_{2}\right)}(b-a)} \mathcal{W}_{a}^{\left(q_{1}, q_{2}-q_{1}\right)}(b), & \text { if } q_{2}>q_{1}, \\
\mathcal{Z}_{a}^{\left(q_{2}, q_{1}-q_{2}\right)}\left(u^{\prime}\right)-\frac{W^{\left(q_{2}\right)}\left(u^{\prime}-a\right)}{W^{\left(q_{2}\right)}(b-a)} \mathcal{Z}_{a}^{\left(q_{2}, q_{1}-q_{2}\right)}(b), & \text { if } q_{1}>q_{2} .\end{cases}
\end{aligned}
$$

When $q_{2}>q_{1}$, substituting it into (4.2) we have, for $a \leq u^{\prime}<b$,

$$
w\left(u^{\prime}\right)=w(a) \frac{\mathcal{W}_{a}^{\left(q_{1}, q_{2}-q_{1}\right)}\left(u^{\prime}\right)}{W^{\left(q_{1}\right)}(a)}-\frac{W^{\left(q_{2}\right)}\left(u^{\prime}-a\right)}{W^{\left(q_{2}\right)}(b-a)}\left[w(a) \frac{\mathcal{W}_{a}^{\left(q_{1}, q_{2}-q_{1}\right)}(b)}{W^{\left(q_{1}\right)}(a)}-w(b)\right] .
$$

To further simplify result (4.3), we let $u^{\prime}=a$. Since $\left\{U^{\prime}(s): s \geq 0\right\}$ has paths of bounded variation, we know $W^{(q)}(0) \neq 0$. From $(4.3)$ we obtain

$$
\frac{w(b)}{\mathcal{W}_{a}^{\left(q_{1}, q_{2}-q_{1}\right)}(b)}=\frac{w(a)}{W^{\left(q_{1}\right)}(a)}
$$

and then (4.3) reduces to

$$
w\left(u^{\prime}\right)=w(b) \frac{\mathcal{W}_{a}^{\left(q_{1}, q_{2}-q_{1}\right)}\left(u^{\prime}\right)}{\mathcal{W}_{a}^{\left(q_{1}, q_{2}-q_{1}\right)}(b)}, \quad a \leq u^{\prime}<b .
$$

Similarly, when $q_{1}>q_{2}$ we obtain

$$
w\left(u^{\prime}\right)=w(b) \frac{\mathcal{Z}_{a}^{\left(q_{2}, q_{1}-q_{2}\right)}\left(u^{\prime}\right)}{\mathcal{Z}_{a}^{\left(q_{2}, q_{1}-q_{2}\right)}(b)}, \quad a \leq u^{\prime}<b .
$$

Notice that both (4.4) and (4.5) are valid for $u^{\prime}<a$ so that they hold for $u^{\prime}<b$.

(3) Finally, for $b \leq u^{\prime} \leq a+b$, when $q_{2}>q_{1}$, we have

$$
w\left(u^{\prime}\right)=\mathbb{E}_{u^{\prime}}\left[e^{-q_{3} \tau} \mathbf{1}_{\left\{\tau<\tau_{b}^{-}\right\}}\right]+\mathbb{E}_{u^{\prime}}\left[e^{-q_{3} \tau_{b}^{-}} w\left(V\left(\tau_{b}^{-}\right)\right) \mathbf{1}_{\left\{\tau_{b}^{-}<\tau\right\}}\right]
$$




$$
=\frac{W^{\left(q_{3}\right)}\left(u^{\prime}-b\right)}{W^{\left(q_{3}\right)}(a)}+\frac{w(b) \mathbb{E}_{u^{\prime}}\left[e^{-q_{3} \tau_{b}^{-}} \mathcal{W}_{a}^{\left(q_{1}, q_{2}-q_{1}\right)}\left(V\left(\tau_{b}^{-}\right)\right) \mathbf{1}_{\left\{\tau_{b}^{-}<\tau\right\}}\right]}{\mathcal{W}_{a}^{\left(q_{1}, q_{2}-q_{1}\right)}(b)} .
$$

In order to determine the expectation in the right-hand side of (4.6), we shall utilize $(2.7)$ by taking $\beta^{(q)}(x)=\mathcal{W}_{a}^{\left(q_{1}, q_{2}-q_{1}\right)}(x)$ where the order of $\mathcal{W}_{a}$ is $q_{1}+q_{2}-q_{1}=q_{2}$. Therefore, we have

$$
\begin{aligned}
& \mathbb{E}_{u^{\prime}}\left[e^{-q_{3} \tau_{b}^{-}} \mathcal{W}_{a}^{\left(q_{1}, q_{2}-q_{1}\right)}\left(V\left(\tau_{b}^{-}\right)\right) \mathbf{1}_{\left\{\tau_{b}^{-}<\tau\right\}}\right] \\
& =\mathcal{W}_{a}^{\left(q_{1}, q_{2}-q_{1}\right)}\left(u^{\prime}\right)-\left(q_{2}-q_{3}\right) \int_{b}^{u^{\prime}} W^{\left(q_{3}\right)}\left(u^{\prime}-z\right) \mathcal{W}_{a}^{\left(q_{1}, q_{2}-q_{1}\right)}(z) d z-\frac{W^{\left(q_{3}\right)}\left(u^{\prime}-b\right)}{W^{\left(q_{3}\right)}(a)} \\
& \times\left[\mathcal{W}_{a}^{\left(q_{1}, q_{2}-q_{1}\right)}(a+b)-\left(q_{2}-q_{3}\right) \int_{b}^{a+b} W^{\left(q_{3}\right)}(a+b-z) \mathcal{W}_{a}^{\left(q_{1}, q_{2}-q_{1}\right)}(z) d z\right] .
\end{aligned}
$$

Let $u^{\prime}=b$. From (4.6) and (4.7) we can get an expression of $w(b)$ :

$$
w(b)=\frac{\mathcal{W}_{a}^{\left(q_{1}, q_{2}-q_{1}\right)}(b)}{\mathcal{W}_{a}^{\left(q_{1}, q_{2}-q_{1}\right)}(a+b)-\left(q_{2}-q_{3}\right) \int_{b}^{a+b} W^{\left(q_{3}\right)}(a+b-z) \mathcal{W}_{a}^{\left(q_{1}, q_{2}-q_{1}\right)}(z) d z} .
$$

Combining results (4.6)-(4.8) gives us, for all $u^{\prime} \leq a+b$ and $q_{2}>q_{1}$,

$$
w\left(u^{\prime}\right)=\frac{w(b)\left[\mathcal{W}_{a}^{\left(q_{1}, q_{2}-q_{1}\right)}\left(u^{\prime}\right)-\left(q_{2}-q_{3}\right) \int_{b}^{u^{\prime}} W^{\left(q_{3}\right)}\left(u^{\prime}-z\right) \mathcal{W}_{a}^{\left(q_{1}, q_{2}-q_{1}\right)}(z) d z\right]}{\mathcal{W}_{a}^{\left(q_{1}, q_{2}-q_{1}\right)}(b)} .
$$

Similarly, when $q_{1}>q_{2}$, we obtain

$$
w\left(u^{\prime}\right)=\frac{w(b)\left[\mathcal{Z}_{a}^{\left(q_{2}, q_{1}-q_{2}\right)}\left(u^{\prime}\right)-\left(q_{2}-q_{3}\right) \int_{b}^{u^{\prime}} W^{\left(q_{3}\right)}\left(u^{\prime}-z\right) \mathcal{Z}_{a}^{\left(q_{2}, q_{1}-q_{2}\right)}(z) d z\right]}{\mathcal{Z}_{a}^{\left(q_{2}, q_{1}-q_{2}\right)}(b)} .
$$

According to the definition of $\bar{m}_{\delta_{1}^{*}, \delta_{2}^{*}, \delta_{3}^{*}}\left(u^{\prime}, a, b\right)$, it can be studied using the spatial homogeneity property of $\left\{U^{\prime}(s): s \geq 0\right\}$ and the above results (4.8) - (4.10). For sufficiently large $m$, when $q_{2}>q_{1}$, we have

$$
\begin{aligned}
& \mathbb{E}_{u^{\prime}}\left[e^{\left.-q_{1} D_{(-\infty, a)}^{*}-q_{2} D_{(a, b)}^{*}-q_{3} D_{(b, a+b)}^{*} \mathbf{1}_{\left\{\tau_{a+b}^{+}<\infty\right\}}\right]}\right. \\
& =\lim _{m \rightarrow \infty} \mathbb{E}_{u^{\prime}}\left[e^{\left.-q_{1} D_{(-m, a)}^{*}-q_{2} D_{(a, b)}^{*}-q_{3} D_{(b, a+b)}^{*} \mathbf{1}_{\left\{\tau_{a+b}^{+}<\tau_{-m}^{-}\right\}}\right]}\right. \\
& =\lim _{m \rightarrow \infty} \frac{\mathcal{W}_{a+m}^{\left(q_{1}, q_{2}-q_{1}\right)}\left(u^{\prime}+m\right)-\left(q_{2}-q_{3}\right) \int_{b+m}^{u^{\prime}+m} W^{\left(q_{3}\right)}\left(u^{\prime}+m-z\right) \mathcal{W}_{a+m}^{\left(q_{1}, q_{2}-q_{1}\right)}(z) d z}{\mathcal{W}_{a+m}^{\left(q_{1}, q_{2}-q_{1}\right)}(a+b+m)-\left(q_{2}-q_{3}\right) \int_{b+m}^{a+b+m} W^{\left(q_{3}\right)}(a+b+m-z) \mathcal{W}_{a+m}^{\left(q_{1}, q_{2}-q_{1}\right)}(z) d z} \\
& =\lim _{m \rightarrow \infty} \frac{\mathcal{W}_{a+m}^{\left(q_{1}, q_{2}-q_{1}\right)}\left(u^{\prime}+m\right)-\left(q_{2}-q_{3}\right) \int_{b}^{u^{\prime}} W^{\left(q_{3}\right)}\left(u^{\prime}-z\right) \mathcal{W}_{a+m}^{\left(q_{1}, q_{2}-q_{1}\right)}(z+m) d z}{\mathcal{W}_{a+m}^{\left(q_{1}, q_{2}-q_{1}\right)}(a+b+m)-\left(q_{2}-q_{3}\right) \int_{b}^{a+b} W^{\left(q_{3}\right)}(a+b-z) \mathcal{W}_{a+m}^{\left(q_{1}, q_{2}-q_{1}\right)}(z+m) d z} .
\end{aligned}
$$


By the dominated convergence theorem and the asymptotic results of $\left\{U^{\prime}(s): s \geq 0\right\}$ given in (2.1) and (2.5), we obtain the following limit

$$
\begin{aligned}
\lim _{m \rightarrow \infty} \frac{\mathcal{W}_{a+m}^{(p, q)}\left(u^{\prime}+m\right)}{W^{(p)}(m)} & =\lim _{m \rightarrow \infty} \frac{W^{(p)}\left(u^{\prime}+m\right)+q \int_{a}^{u^{\prime}} W^{(p+q)}\left(u^{\prime}-y\right) W^{(p)}(y+m) d y}{W^{(p)}(m)} \\
& =e^{\rho(p) u^{\prime}}+q \int_{a}^{u^{\prime}} W^{(p+q)}\left(u^{\prime}-y\right) e^{\rho(p) y} d y \\
& =e^{\rho(p) a} \mathcal{H}^{(p, q)}\left(u^{\prime}-a\right) .
\end{aligned}
$$

Using (4.12), (4.11) can be further simplified as

$$
\begin{aligned}
& \mathbb{E}_{u^{\prime}}\left[e^{-q_{1} D_{(-\infty, a)}^{*}-q_{2} D_{(a, b)}^{*}-q_{3} D_{(b, a+b)}^{*}} \mathbf{1}_{\left\{\tau_{a+b}^{+}<\infty\right\}}\right] \\
& =\frac{\mathcal{H}^{\left(q_{1}, q_{2}-q_{1}\right)}\left(u^{\prime}-a\right)-\left(q_{2}-q_{3}\right) \int_{b}^{u^{\prime}} W^{\left(q_{3}\right)}\left(u^{\prime}-z\right) \mathcal{H}^{\left(q_{1}, q_{2}-q_{1}\right)}(z-a) d z}{\mathcal{H}^{\left(q_{1}, q_{2}-q_{1}\right)}(b)-\left(q_{2}-q_{3}\right) \int_{b}^{a+b} W^{\left(p+q_{3}\right)}(a+b-z) \mathcal{H}^{\left(q_{1}, q_{2}-q_{1}\right)}(z-a) d z} .
\end{aligned}
$$

Similarly, when $q_{1}>q_{2}$, we can obtain

$$
\begin{aligned}
& \mathbb{E}_{u^{\prime}}\left[e^{-q_{1} D_{(-\infty, a)}^{*}-q_{2} D_{(a, b)}^{*}-q_{3} D_{(b, a+b)}^{*}} \mathbf{1}_{\left\{\tau_{a+b}^{+}<\infty\right\}}\right] \\
& =\lim _{m \rightarrow \infty} \frac{\mathcal{Z}_{a+m}^{\left(q_{2}, q_{1}-q_{2}\right)}\left(u^{\prime}+m\right)-\left(q_{2}-q_{3}\right) \int_{b}^{u^{\prime}} W^{\left(q_{3}\right)}\left(u^{\prime}-z\right) \mathcal{Z}_{a+m}^{\left(q_{2}, q_{1}-q_{2}\right)}(z+m) d z}{\mathcal{Z}_{a+m}^{\left(q_{2}, q_{1}-q_{2}\right)}(a+b+m)-\left(q_{2}-q_{3}\right) \int_{b}^{a+b} W^{\left(q_{3}\right)}(a+b-z) \mathcal{Z}_{a+m}^{\left(q_{2}, q_{1}-q_{2}\right)}(z+m) d z},
\end{aligned}
$$

and

$$
\begin{aligned}
\lim _{m \rightarrow \infty} \frac{\mathcal{Z}_{a+m}^{(p, q)}\left(u^{\prime}+m\right)}{W^{(p+q)}(m)} & =\lim _{m \rightarrow \infty} \frac{W^{(p+q)}\left(u^{\prime}+m\right)-q \int_{a}^{u^{\prime}} W^{(p+q)}(y+m) W^{(p)}\left(u^{\prime}-y\right) d y}{W^{(p+q)}(m)} \\
& =e^{\rho(p+q) u^{\prime}}-q \int_{a}^{u^{\prime}} e^{\rho(p+q) y} W^{(p)}\left(u^{\prime}-y\right) d y \\
& =e^{\rho(p+q) a} e^{\rho(p+q)\left(u^{\prime}-a\right)}\left[1-q \int_{0}^{u^{\prime}-a} e^{\rho(p+q)(-x)} W^{(p)}(x) d x\right] \\
& =e^{\rho(p+q) a} \mathcal{H}^{(p+q,-q)}\left(u^{\prime}-a\right) .
\end{aligned}
$$

(4.14) and (4.15) give the same result (4.13). Finally, let $q_{i}=\delta_{i}^{*}, i=1,2,3$, then Theorem 3 is proved.

\section{Remarks:}

- Theorem 3 generalizes Corollary 2 in [30];

- The expression in Theorem 3 is given in terms of scale functions $W^{(p)}$ and $\mathcal{H}^{(p, q)}$ which can be computed by Laplace inversion. Several numerical methods for dealing with Laplace inversion of the scale functions can be found in [17]. 


\section{Sparre Andersen risk model in the presence of a constant dividend barrier}

In this section, we shall introduce a constant dividend barrier into our original Sparre Andersen risk model (1.1). Let $a=0$, and $b>u$ which is the dividend barrier. As long as the surplus level goes beyond $b$, dividends are paid out continuously at rate $c$ until the next claim occurs. A sample path of the new model is given below:

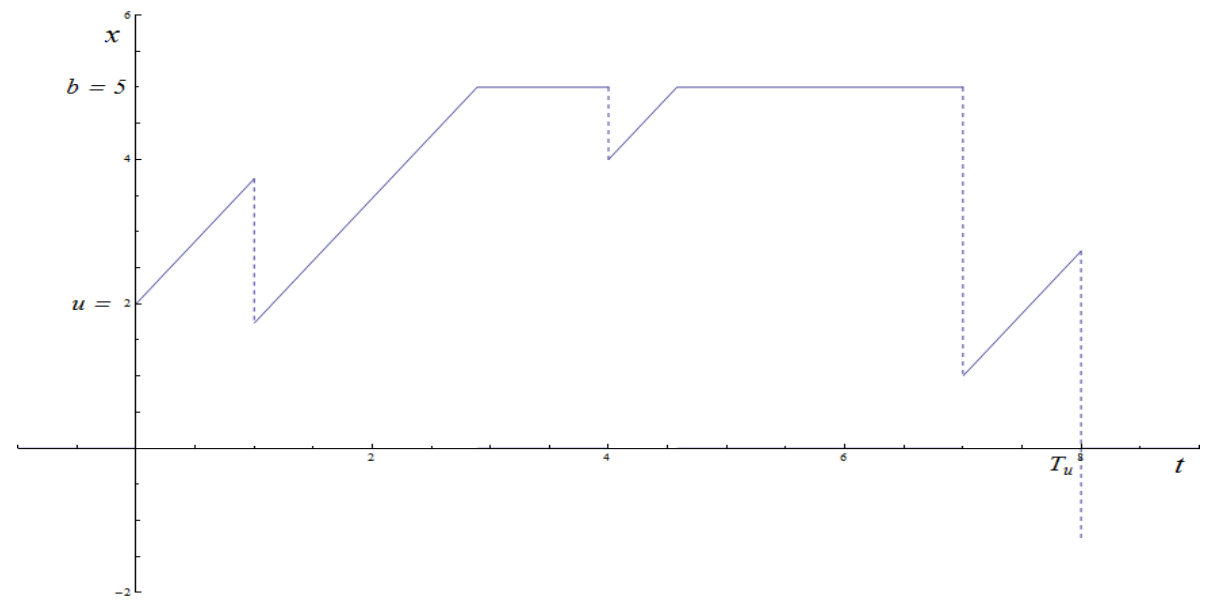

Figure 3: A sample path of $\left\{U_{b}(t): t \geq 0\right\}$

In this renewal risk model with the presence of a constant dividend barrier, we shall derive the Laplace transforms of the time of ruin and the non-dividend duration.

Let $\left\{U_{b}(t): t \geq 0\right\}$ denote the surplus level at time $t$ of this new Sparre Andersen surplus process with an initial surplus $U_{b}(0)=u \geq 0$, then we have

$$
d U_{b}(t)= \begin{cases}c d t-d U(t), & U_{b}(t)<b \\ -d U(t), & U_{b}(t) \geq b\end{cases}
$$

The time of ruin in this model is defined as

$$
T_{u}^{b}=\inf \left\{t>0: U_{b}(t)<0 \mid U_{b}(0)=u\right\}, \quad u \geq 0,
$$

and the ultimate probability of ruin is $\psi^{b}(u)=\mathbb{P}\left(T_{u}^{b}<\infty\right) \equiv 1$. For $\delta \geq 0$, the Laplace transform of $T_{u}^{b}$ is denoted by

$$
m_{\delta}^{b}(u)=\mathbb{E}\left[e^{-\delta T_{u}^{b}}\right], \quad u \geq 0 .
$$

The total time until ruin $T_{u}^{b}$ can be decomposed into two parts:

$$
T_{u}^{b}=\int_{0}^{T_{u}^{b}} \mathbf{1}_{\left\{0<U_{b}(t)<b\right\}} d t+\int_{0}^{T_{u}^{b}} \mathbf{1}_{\left\{U_{b}(t)=b\right\}} d t=D_{0}+D_{1},
$$


where $D_{1}$ denotes the dividend paying duration prior to ruin and $D_{0}$ denotes the corresponding non-dividend paying duration. In order to study $D_{0}$ and $D_{1}$, we shall perform the same three-step transformation as given in Section 3. A sample path of the final transformed process, denoted by $\left\{U_{b}^{\prime}(s): s \geq 0\right\}$, is given below



Figure 4: A sample path of $\left\{U_{b}^{\prime}(s): s \geq 0\right\}$

In the $(s, z)$ system of coordinates, $\left\{U_{b}^{\prime}(s): s \geq 0\right\}$ can be expressed as

$$
U_{b}^{\prime}(s)= \begin{cases}u^{\prime}+s-c \sum_{i=1}^{N_{s}^{*}} K_{i}, & s<\zeta_{1}, \\ s-\sigma_{n}-c \sum_{i=1}^{N_{s-\sigma_{n}}^{*}} K_{i}, & \sigma_{n} \leq s<\zeta_{n+1}, \\ 0, & \zeta_{n} \leq s<\sigma_{n},\end{cases}
$$

with $\left\{\zeta_{n}\right\}$ and $\left\{\sigma_{n}\right\}, n \in \mathbb{N}^{+}$, defined as follows:

$$
\left\{\begin{array}{l}
\zeta_{1}=\inf \left\{s>0: U_{b}^{\prime}(s)=0\right\} \\
\sigma_{1}=\inf \left\{s>\zeta_{1}: 0<U_{b}^{\prime}(s)\right\} \\
\zeta_{n+1}=\inf \left\{s>\sigma_{n}: U_{b}^{\prime}(s)=0\right\} \\
\sigma_{n+1}=\inf \left\{s>\zeta_{n+1}: 0<U_{b}^{\prime}(s)\right\}
\end{array}\right.
$$

and $u^{\prime}=b-u-c \xi$ where $\xi$ is the value of $K_{1}$. It is worth noting that $\left\{\zeta_{n}\right\}_{n \in \mathbb{N}^{+}}$are i.i.d. and follow exponential distribution with parameter $\lambda$. The first passage time $\tau_{b}$, defined as the first time that $\left\{U_{b}^{\prime}(s): s \geq 0\right\}$ up-crosses $b$, has the following definition

$$
\tau_{b}=\inf \left\{s>0: U_{b}^{\prime}(s)>b\right\},
$$

which has a similar relationship with $T_{u}^{b}$ as given in (3.4):

$$
T_{u}^{b}=\left(\tau_{b}-u\right) / c .
$$


The Laplace transform of $\tau_{b}$ is defined as

$$
\bar{m}_{\delta^{*}}^{b}\left(u^{\prime}\right)=\mathbb{E}_{u^{\prime}}\left[e^{-\delta^{*} \tau_{b}} \mathbf{1}_{\left\{\tau_{b}<\infty\right\}}\right], \quad u^{\prime}<b .
$$

We can prove the same relationship between the two Laplace transforms, $m_{\delta}^{b}(u)$ and $\bar{m}_{\delta^{*}}^{b}\left(u^{\prime}\right)$, as the one given in Theorem 1:

$$
m_{\delta}^{b}(u)=\int_{0}^{\infty} h_{0}(\xi) e^{\delta^{*} u} \bar{m}_{\delta^{*}}^{b}\left(u^{\prime}\right) d \xi
$$

where $\delta^{*}=\delta / c$. Therefore, it suffices to find $\bar{m}_{\delta^{*}}^{b}\left(u^{\prime}\right)$ in order to determine $m_{\delta}^{b}(u)$. Now we decompose $\tau_{b}$ as

$$
\tau_{b}=\int_{0}^{\tau_{b}} \mathbf{1}_{\left\{0<U_{b}^{\prime}(s)<b\right\}} d s+\int_{0}^{\tau_{b}} \mathbf{1}_{\left\{U_{b}^{\prime}(s)=0\right\}} d s=D_{0}^{*}+D_{1}^{*},
$$

where $\left(D_{0}^{*}, D_{1}^{*}\right)$ and $\left(D_{0}, D_{1}\right)$ satisfy the following relationship

$$
D_{0}^{*}=c D_{0}+u, \quad D_{1}^{*}=c D_{1} .
$$

The Laplace transforms of the occupation times are defined in the following

$$
\begin{aligned}
m_{b, i}(u) & =\mathbb{E}\left[e^{-\delta_{i} D_{i}} \mathbf{1}_{\left\{T_{u}^{b}<\infty\right\}}\right], \quad i=0,1, \\
\bar{m}_{b, i}\left(u^{\prime}\right) & =\mathbb{E}_{u^{\prime}}\left[e^{-\delta_{i}^{*} D_{i}^{*}} \mathbf{1}_{\left\{\tau_{b}<\infty\right\}}\right], \quad i=0,1,
\end{aligned}
$$

and they satisfy the following results

$$
\begin{aligned}
& m_{b, 0}(u)=\int_{0}^{\infty} h_{0}(\xi) e^{\delta_{0}^{*} u} \bar{m}_{b, 0}\left(u^{\prime}\right) d \xi \\
& m_{b, 1}(u)=\int_{0}^{\infty} h_{0}(\xi) \bar{m}_{b, 1}\left(u^{\prime}\right) d \xi
\end{aligned}
$$

It is sufficient to find $\bar{m}_{b, 0}\left(u^{\prime}\right)$ and $\bar{m}_{b, 1}\left(u^{\prime}\right)$ in the transformed process $\left\{U_{b}^{\prime}(s): s \geq 0\right\}$. In the following subsections, we shall derive explicit expressions of $\bar{m}_{\delta^{*}}^{b}\left(u^{\prime}\right)$ and $\bar{m}_{b, 0}\left(u^{\prime}\right)$.

\subsection{Laplace transform of first passage time $\tau_{b}$}

In this subsection, we shall prove the following explicit result of $\bar{m}_{\delta^{*}}^{b}\left(u^{\prime}\right)$ :

Theorem 4 The Laplace transform of the first passage time $\tau_{b}$ of the process $\left\{U_{b}^{\prime}(s): s \geq 0\right\}$ can be expressed as

$$
\bar{m}_{\delta^{*}}^{b}\left(u^{\prime}\right)=\frac{\sum_{n=0}^{\infty} \lambda^{n+1}\left(\mathcal{T}_{\rho^{*}} h\right)^{* n} * \mathcal{T}_{\rho^{*}} \mathcal{T}_{\delta^{*}} h\left(u^{\prime}\right)+\left[1-\lambda \mathcal{T}_{\rho^{*}} \mathcal{T}_{\delta^{*}} h(0)\right] W^{\left(\delta^{*}\right)}\left(u^{\prime}\right)}{\left[1-\lambda \mathcal{T}_{\rho^{*}} \mathcal{T}_{\delta^{*}} h(0)\right] W^{\left(\delta^{*}\right)}(b)+\sum_{n=0}^{\infty} \lambda^{n+1}\left(\mathcal{T}_{\rho^{*}} h\right)^{* n} * \mathcal{T}_{\rho^{*}} \mathcal{T}_{\delta^{*}} h(b)}, \quad 0 \leq u^{\prime} \leq b,
$$

and

$$
\bar{m}_{\delta^{*}}^{b}\left(u^{\prime}\right)=e^{\delta^{*} u^{\prime}} \bar{m}_{\delta^{*}}^{b}(0), \quad u^{\prime}<0,
$$


where $\rho^{*}$ is the non-negative solution of the Lundberg fundamental equation corresponding to $\delta^{*}, \mathcal{T}$ is the Dickson-Hipp operator with definition

$$
\mathcal{T}_{s} h(x)=\int_{x}^{\infty} e^{-s(y-x)} h(y) d y
$$

and the scale function $W^{\left(\delta^{*}\right)}\left(u^{\prime}\right)$ can be expressed as

$$
W^{\left(\delta^{*}\right)}\left(u^{\prime}\right)=e^{\rho^{*} u^{\prime}} \sum_{n=0}^{\infty} \lambda^{n} \hat{h}\left(\rho^{*}\right)^{n} \int_{0}^{u^{\prime}} \bar{H}^{* n}(x ; \rho) d x, \quad u^{\prime}>0,
$$

with $W^{\left(\delta^{*}\right)}(0)=1$. Furthermore, $h\left(x ; \rho^{*}\right)$ is the Esscher transform of $h(x)$ with definition

$$
h\left(x ; \rho^{*}\right)=\frac{e^{-\rho^{*} x} h(x)}{\hat{h}\left(\rho^{*}\right)},
$$

and $\bar{H}\left(x ; \rho^{*}\right)=\int_{x}^{\infty} h\left(y ; \rho^{*}\right) d y$.

Proof. We shall employ the integro-differential equation (IDE) approach to prove Theorem 4. Thanks to the renewal property of the Poisson process, we obtain an IDE of $\bar{m}_{\delta^{*}}^{b}\left(u^{\prime}\right)$ using the infinitesimal technique over a small time interval:

$$
\begin{aligned}
\frac{\partial}{\partial u^{\prime}} \bar{m}_{\delta^{*}}^{b}\left(u^{\prime}\right)= & \left(\lambda+\delta^{*}\right) \bar{m}_{\delta^{*}}^{b}\left(u^{\prime}\right)-\lambda \int_{0}^{u^{\prime}} \bar{m}_{\delta^{*}}^{b}\left(u^{\prime}-x\right) h(x) d x \\
& -\lambda e^{\delta^{*} u^{\prime}} \bar{m}_{\delta^{*}}^{b}(0) \int_{u^{\prime}}^{\infty} e^{-\delta^{*} x} h(x) d x,
\end{aligned}
$$

with a boundary condition $\bar{m}_{\delta^{*}}^{b}(b)=1$. It follows from the general theory of differential equations that every solution of this non-homogeneous IDE is of the form (see [29] for example)

$$
\bar{m}_{\delta^{*}}^{b}\left(u^{\prime}\right)=g\left(u^{\prime}\right)+C W^{\left(\delta^{*}\right)}\left(u^{\prime}\right), \quad 0 \leq u^{\prime}<\infty,
$$

where $C$ is a constant and the scale function $W^{\left(\delta^{*}\right)}$ satisfies the homogeneous IDE:

$$
\frac{d}{d u^{\prime}} W^{\left(\delta^{*}\right)}\left(u^{\prime}\right)=\left(\lambda+\delta^{*}\right) W^{\left(\delta^{*}\right)}\left(u^{\prime}\right)-\lambda \int_{0}^{u^{\prime}} W^{\left(\delta^{*}\right)}\left(u^{\prime}-x\right) g(x) d x, \quad 0 \leq u^{\prime}<\infty,
$$

with an initial condition $W^{\left(\delta^{*}\right)}(0)=1$. The function $g\left(u^{\prime}\right)$ is a particular solution to the following IDE, for $0 \leq u^{\prime}<\infty$,

$$
\frac{d}{d u^{\prime}} g\left(u^{\prime}\right)=\left(\lambda+\delta^{*}\right) g\left(u^{\prime}\right)-\lambda \int_{0}^{u^{\prime}} g\left(u^{\prime}-x\right) h(x) d x-\lambda e^{\delta^{*} u^{\prime}} \bar{m}_{\delta^{*}}^{b}(0) \int_{u^{\prime}}^{\infty} e^{-\delta^{*} x} h(x) d x .
$$

Using the boundary condition of $\bar{m}_{\delta^{*}}^{b}(b)$, we obtain

$$
C=\frac{1-g(b)}{W^{\left(\delta^{*}\right)}(b)},
$$


and then the solution for $\bar{m}_{\delta^{*}}^{b}\left(u^{\prime}\right)$ becomes

$$
\bar{m}_{\delta^{*}}^{b}\left(u^{\prime}\right)=g\left(u^{\prime}\right)+\frac{1-g(b)}{W^{\left(\delta^{*}\right)}(b)} W^{\left(\delta^{*}\right)}\left(u^{\prime}\right), \quad 0 \leq u^{\prime} \leq b .
$$

By the definition of $\bar{m}_{\delta^{*}}^{b}\left(u^{\prime}\right)$, we know $\bar{m}_{\delta^{*}}^{b}(0) \neq 0$. Dividing by $\bar{m}_{\delta^{*}}^{b}(0)$ on both sides of (5.10) and letting $\bar{g}\left(u^{\prime}\right)=\frac{g\left(u^{\prime}\right)}{\bar{m}_{\delta^{*}}^{b}(0)},(5.10)$ can be rewritten as, for $0 \leq u^{\prime}<\infty$,

$$
\frac{d}{d u^{\prime}} \bar{g}\left(u^{\prime}\right)=\left(\lambda+\delta^{*}\right) \bar{g}\left(u^{\prime}\right)-\lambda \int_{0}^{u^{\prime}} \bar{g}\left(u^{\prime}-x\right) h(x) d x-\lambda e^{\delta^{*} u^{\prime}} \int_{u^{\prime}}^{\infty} e^{-\delta^{*} x} h(x) d x .
$$

Taking Laplace transform on both sides of (5.12) gives

$$
\hat{\bar{g}}(s)=\frac{\bar{g}(0)-\lambda \mathcal{T}_{s} \mathcal{T}_{\delta^{*}} h(0)}{s-\left(\lambda+\delta^{*}\right)+\lambda \hat{h}(s)} .
$$

where $\mathcal{T}$ is the Dickson-Hipp operator and properties of this translation operator can be found in [15] and [25]. Recall that for $\delta^{*} \geq 0$ and $s \geq 0$, the corresponding Lundberg fundamental equation under the transformed surplus process $\left\{U_{b}^{\prime}(s): s \geq 0\right\}$ is

$$
s-\left(\lambda+\delta^{*}\right)+\lambda \hat{h}(s)=0
$$

which has one unique non-negative root $\rho^{*}$. When $s=\rho^{*}$ the numerator should also equal 0 , i.e.

$$
\bar{g}(0)=\lambda \mathcal{T}_{\rho^{*}} \mathcal{T}_{\delta^{*}} h(0)
$$

Therefore, (5.13) can be further expressed as

$$
\hat{\bar{g}}(s)=\frac{\lambda \mathcal{T}_{s} \mathcal{T}_{\rho^{*}} \mathcal{T}_{\delta^{*}} h(0)}{1-\lambda \mathcal{T}_{s} \mathcal{T}_{\rho^{*}} h(0)} .
$$

By inverting (5.14), we obtain an expression for $\bar{g}\left(u^{\prime}\right)$ as

$$
\bar{g}\left(u^{\prime}\right)=\sum_{n=0}^{\infty} \lambda^{n+1}\left(\mathcal{T}_{\rho} h\right)^{* n} * \mathcal{T}_{\rho} \mathcal{T}_{\delta^{*}} h\left(u^{\prime}\right),
$$

where $a * b$ denotes the convolution of functions $a$ and $b$ and $a^{* n}$ denotes the $n$-th fold convolution of the function $a$. Thus we obtain an expression of $g\left(u^{\prime}\right)$

$$
g\left(u^{\prime}\right)=\bar{m}_{\delta^{*}}^{b}(0) \sum_{n=0}^{\infty} \lambda^{n+1}\left(\mathcal{T}_{\rho^{*}} h\right)^{* n} * \mathcal{T}_{\rho^{*}} \mathcal{T}_{\delta^{*}} h\left(u^{\prime}\right), \quad 0 \leq u^{\prime}<\infty .
$$

In order to determine $\bar{m}_{\delta^{*}}^{b}(0)$, letting $u^{\prime}=0$ in (5.11) yields

$$
\bar{m}_{\delta^{*}}^{b}(0)=\bar{m}_{\delta^{*}}^{b}(0) \lambda \mathcal{T}_{\rho} \mathcal{T}_{\delta^{*}} h(0)+\frac{1-g(b)}{W^{\left(\delta^{*}\right)}(b)}
$$




$$
=\bar{m}_{\delta^{*}}^{b}(0) \lambda \mathcal{T}_{\rho} \mathcal{T}_{\delta^{*}} h(0)+\frac{1-\bar{m}_{\delta^{*}}^{b}(0) \sum_{n=0}^{\infty} \lambda^{n+1}\left(\mathcal{T}_{\rho} h\right)^{* n} * \mathcal{T}_{\rho} \mathcal{T}_{\delta^{*}} h(b)}{W^{\left(\delta^{*}\right)}(b)} .
$$

Solving equation (5.16) gives

$$
\bar{m}_{\delta^{*}}^{b}(0)=\frac{1}{W^{\left(\delta^{*}\right)}(b)\left[1-\lambda \mathcal{T}_{\rho^{*}} \mathcal{T}_{\delta^{*}} h(0)\right]+\sum_{n=0}^{\infty} \lambda^{n+1}\left(\mathcal{T}_{\rho^{*}} h\right)^{* n} * \mathcal{T}_{\rho^{*}} \mathcal{T}_{\delta^{*}} h(b)} .
$$

Substituting (5.15) and (5.17) into (5.11), we obtain (5.5). We shall then derive the explicit expression for $W^{\left(\delta^{*}\right)}\left(u^{\prime}\right)$ given in (5.7). Following the methodology used in [29], we introduce a function $\Phi\left(u^{\prime}\right), 0<u^{\prime}<\infty$, such that

$$
W^{\left(\delta^{*}\right)}\left(u^{\prime}\right)=\frac{\Phi\left(u^{\prime}\right)}{\Phi(0)} e^{\rho^{*} u^{\prime}}
$$

where $\Phi\left(u^{\prime}\right)$ satisfies the following IDE

$$
\begin{aligned}
\frac{d}{d u^{\prime}} \Phi\left(u^{\prime}\right) & =-\lambda \int_{0}^{u^{\prime}} \Phi\left(u^{\prime}-x\right) e^{-\rho^{*} x} h(x) d x-\left[\rho^{*}-\left(\lambda+\delta^{*}\right)\right] \Phi\left(u^{\prime}\right) \\
& =-\lambda \int_{0}^{u^{\prime}} \Phi\left(u^{\prime}-x\right) e^{-\rho^{*} x} h(x) d x+\lambda \hat{h}\left(\rho^{*}\right) \Phi\left(u^{\prime}\right)
\end{aligned}
$$

and

$$
\Phi(0)=1-\lambda \hat{h}\left(\rho^{*}\right) \int_{0}^{\infty} x h\left(x ; \rho^{*}\right) d x
$$

where $h\left(x ; \rho^{*}\right)$ is the Esscher transform of $h(x)$. Using the above symbols, (5.19) can be re-written as

$$
\frac{d}{d u^{\prime}} \Phi\left(u^{\prime}\right)=\lambda \hat{h}\left(\rho^{*}\right)\left[\Phi\left(u^{\prime}\right)-\int_{0}^{u^{\prime}} \Phi\left(u^{\prime}-x\right) h\left(x ; \rho^{*}\right) d x\right], \quad 0<u^{\prime}<\infty .
$$

Therefore $\Phi\left(u^{\prime}\right)$ is the tail of a compound geometric distribution with geometric parameter $\Phi(0)$ and claim size distribution which has p.d.f. $\bar{H}\left(x ; \rho^{*}\right) / \int_{0}^{\infty} x h\left(x ; \rho^{*}\right) d x$, where $\bar{H}\left(x ; \rho^{*}\right)=\int_{x}^{\infty} h\left(y ; \rho^{*}\right) d y$ and $\hat{\bar{H}}\left(s ; \rho^{*}\right)$ is its Laplace transform.

Otherwise taking Laplace transforms on both sides of (5.20) and rearranging terms yields

$$
\hat{\Phi}(s)=\frac{\Phi(0)}{s-\lambda \hat{h}\left(\rho^{*}\right)\left[1-\hat{h}\left(s ; \rho^{*}\right)\right]}=\frac{\Phi(0)}{s\left[1-\lambda \hat{h}\left(\rho^{*}\right) \hat{\bar{H}}\left(s ; \rho^{*}\right)\right]},
$$

which can be further written as

$$
\hat{\Phi}(s)=\frac{\Phi(0)}{s} \sum_{n=0}^{\infty}\left(\lambda \hat{h}\left(\rho^{*}\right) \hat{\bar{H}}\left(s ; \rho^{*}\right)\right)^{n} .
$$

Inverting the Laplace transforms on both sides of the above equation with respect to $s$ gives us

$$
\Phi\left(u^{\prime}\right)=\Phi(0) \sum_{n=0}^{\infty}\left(\lambda \hat{h}\left(\rho^{*}\right)\right)^{n} \int_{0}^{u^{\prime}} \bar{H}^{* n}\left(x ; \rho^{*}\right) d x
$$


hence by substituting (5.21) into (5.18) we obtain (5.7).

Note that within the above derivations we only considered the case of $u^{\prime} \geq 0$, which means that the first claim comes before the surplus reaches $b$ in the original risk process $\{U(t): t \geq 0\}$, i.e. $u+c K_{1} \leq b$. When the surplus reaches $b$ before the first claim comes, i.e. $u+c K_{1}>b$ or $u^{\prime}<0$, we can see the following sample path:

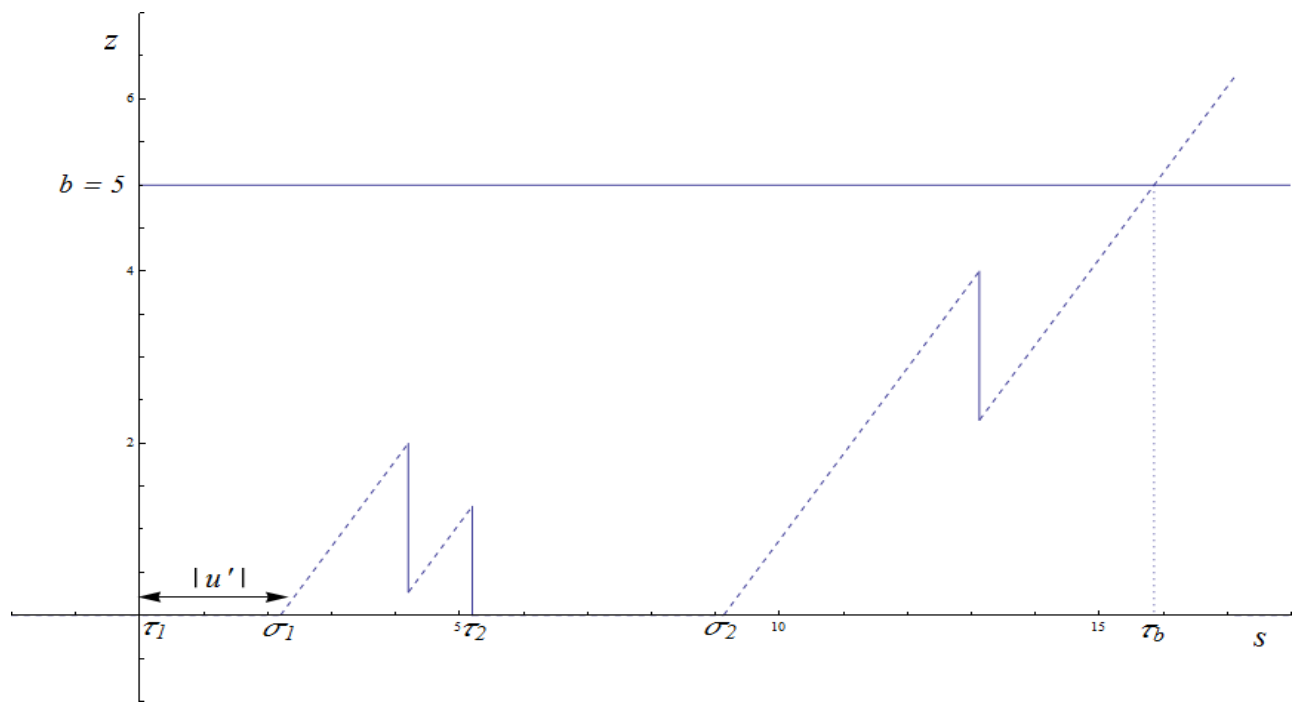

Figure 5: A sample path of $\left\{U_{b}^{\prime}(s): s \geq 0\right\}$ with $u^{\prime}<0$

In this case, the definition of our surplus process $\left\{U_{b}^{\prime}(s): s \geq 0\right\}$ needs to change such that $\zeta_{1}=0$ and $\sigma_{1}-\zeta_{1}=u^{\prime}$. As $\left\{U_{b}^{\prime}(s): s \geq 0\right\}$ starts and remains at 0 for a period of $\left|u^{\prime}\right|$ until the income arrives, $\bar{m}_{\delta^{*}}^{b}\left(u^{\prime}\right)$ can be expressed in terms of $\bar{m}_{\delta^{*}}^{b}(0)$ as given in (5.6). Thus Theorem 4 is proved.

\subsection{Laplace transform of occupation time $D_{0}^{*}$}

In this section, we derive the Laplace transform of $D_{0}^{*}$, which can be used to study the nondividend paying duration $D_{0}$ of our model (1.1), and express it in terms of scale functions. Our result is given as follows.

Theorem 5 The Laplace transform of $D_{0}^{*}$ given the initial surplus $U_{b}^{\prime}(0)=u^{\prime}$ is

$$
\mathbb{E}_{u^{\prime}}\left[e^{-\delta_{0}^{*} D_{0}^{*}} \mathbf{1}_{\left\{\tau_{b}<\infty\right\}}\right]=\frac{Z^{\left(\delta_{0}^{*}\right)}\left(u^{\prime}\right)}{Z^{\left(\delta_{0}^{*}\right)}(b)}, \quad u^{\prime} \in \mathbb{R} .
$$

Proof. One can see from Figure 6 that the process $\left\{U_{b}^{\prime}(s): s \geq 0\right\}$ is a combination of several compound Poisson processes with some delay periods in between up until the first hitting time $\tau_{b}$. In other words, $\left\{U_{b}^{\prime}(s): s \geq 0\right\}$ stays in $(0, b)$ several times (compound Poisson processes) and stays at 0 in between until time $\tau_{b}$. Since we are only interested in 
the total occupation time that $\left\{U_{b}^{\prime}(s): s \geq 0\right\}$ stays in $(0, b)$ before $\tau_{b}$, i.e. $D_{0}^{*}$, we can just extract the delay periods from the process $\left\{U_{b}^{\prime}(s): s \geq 0\right\}$ and then the remaining process only contain the compound Poisson pieces. This new process can be modeled by

$$
\bar{U}_{b}^{\prime}(s)=U^{\prime}(s)-\min \left(0, \inf _{0<t<s} U^{\prime}(t)\right), \quad s \geq 0,
$$

and the first passage time of level $b$ for this process has the definition

$$
r_{b}=\inf \left\{s>0: \bar{U}_{b}^{\prime}(s)>b\right\}=\inf \left\{s>0: U^{\prime}(s)>b\right\} .
$$

One can see that the process $\bar{U}_{b}^{\prime}(s)$ is the process $\left\{U^{\prime}(s): s \geq 0\right\}$ reflected on the boundary $z=0$, which is shown in Figure 6 .

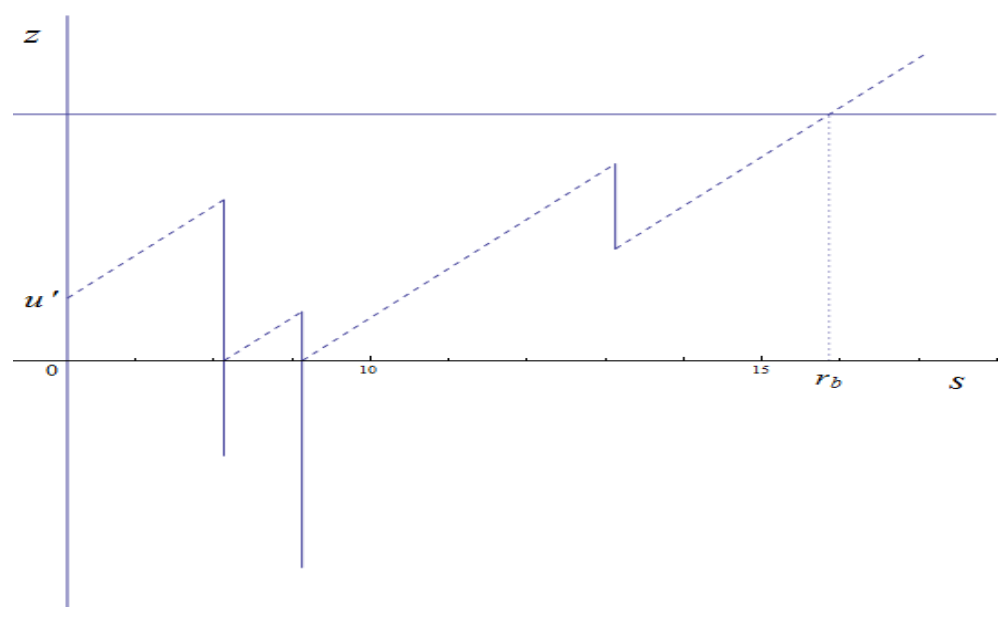

Figure 6: A sample path of $\left\{\bar{U}_{b}^{\prime}(s): s \geq 0\right\}$

Thus our problem of studying $D_{0}^{*}$ becomes one regarding $r_{b}$, i.e.

$$
\mathbb{E}_{u^{\prime}}\left[e^{-\delta_{0}^{*} D_{0}^{*}} \mathbf{1}_{\left\{\tau_{b}<\infty\right\}}\right]=\mathbb{E}_{u^{\prime}}\left[e^{-\delta_{0}^{*} r_{b}} \mathbf{1}_{\left\{r_{b}<\infty\right\}}\right]
$$

Define

$$
r_{0}=\inf \left\{s>0: U^{\prime}(s)<0\right\},
$$

and we know $r_{0}<\infty$ and $\lim _{b \rightarrow 0} r_{b} \neq r_{0}$.

(1) If $0 \leq u^{\prime} \leq b$, by taking into account the memoryless property of the exponential distribution, the Laplace transform of $r_{b}$ conditioned on $\bar{U}_{b}^{\prime}(0)=u^{\prime}$ can be expressed as

$$
\begin{aligned}
& \mathbb{E}_{u^{\prime}}\left[e^{-\delta_{0}^{*} r_{b}} \mathbf{1}_{\left\{r_{b}<\infty\right\}}\right] \\
& =\mathbb{E}_{u^{\prime}}\left[e^{-\delta_{0}^{*} r_{b}} \mathbf{1}_{\left\{r_{b}<r_{0}\right\}}\right]+\mathbb{E}_{u^{\prime}}\left[e^{-\delta_{0}^{*} r_{0}} \mathbf{1}_{\left\{r_{0}<r_{b}\right\}}\right] \mathbb{E}_{0}\left[e^{-\delta_{0}^{*} r_{b}} \mathbf{1}_{\left\{r_{b}<\infty\right\}}\right] \\
& =\frac{W^{\left(\delta_{0}^{*}\right)}\left(u^{\prime}\right)}{W^{\left(\delta_{0}^{*}\right)}(b)}+\left[Z^{\left(\delta_{0}^{*}\right)}\left(u^{\prime}\right)-W^{\left(\delta_{0}^{*}\right)}\left(u^{\prime}\right) \frac{Z^{\left(\delta_{0}^{*}\right)}(b)}{W^{\left(\delta_{0}^{*}\right)}(b)}\right] \mathbb{E}_{0}\left[e^{-\delta_{0}^{*} r_{b}} \mathbf{1}_{\left\{r_{b}<\infty\right\}}\right] .
\end{aligned}
$$


By the Initial Value Theorem, we have

$$
\lim _{\lambda \rightarrow+\infty} \int_{0}^{\infty} e^{-\lambda x} W^{\left(\delta_{0}^{*}\right)}(x) d x=W^{\left(\delta_{0}^{*}\right)}(0),
$$

and from the definition of the scale function and the definition of $\Psi$, we know $W^{\left(\delta_{0}^{*}\right)}(0)=1$ and $Z^{\left(\delta_{0}^{*}\right)}(0)=1$. Then, setting $u^{\prime}=0$ in (5.23) yields

$$
\mathbb{E}_{0}\left[e^{-\delta_{0}^{*} r_{b}} \mathbf{1}_{\left\{r_{b}<\infty\right\}}\right]=\frac{1}{W^{\left(\delta_{0}^{*}\right)}(b)}+\left[1-\frac{Z^{\left(\delta_{0}^{*}\right)}(b)}{W^{\left(\delta_{0}^{*}\right)}(b)}\right] \mathbb{E}_{0}\left[e^{-\delta_{0}^{*} r_{b}} \mathbf{1}_{\left\{r_{b}<\infty\right\}}\right],
$$

and hence

$$
\mathbb{E}_{0}\left[e^{-\delta_{0}^{*} r_{b}} \mathbf{1}_{\left\{r_{b}<\infty\right\}}\right]=\frac{1}{Z^{\left(\delta_{0}^{*}\right)}(b)}
$$

Substituting (5.24) into (5.23), we obtain, for $0 \leq u^{\prime} \leq b$,

$$
\mathbb{E}_{u^{\prime}}\left[e^{-\delta_{0}^{*} r_{b}} \mathbf{1}_{\left\{r_{b}<\infty\right\}}\right]=\frac{Z^{\left(\delta_{0}^{*}\right)}\left(u^{\prime}\right)}{Z^{\left(\delta_{0}^{*}\right)}(b)} .
$$

The result (5.22) is proved.

(2) For the case $u^{\prime}<0$, similar to the previous subsection, $\left\{U_{b}^{\prime}(s): s \geq 0\right\}$ in Figure 5 starts from $s=0$ and stays at $z=0$ for $\left|u^{\prime}\right|$ time units then increases with rate 1 . In Figure 5 , no matter how negative $u^{\prime}$ is, $\left\{\bar{U}_{b}^{\prime}(s): s \geq 0\right\}$ always starts with 0 since the whole $\left|u^{\prime}\right|$ contributes to the delay period, or $D_{1}^{*}$. Therefore, for $u^{\prime}<0$,

$$
\begin{aligned}
\mathbb{E}\left[e^{-\delta_{0}^{*} D_{0}^{*}} \mathbf{1}_{\left\{\tau_{b}<\infty\right\}} \mid U_{b}^{\prime}(0)=u^{\prime}\right] & =\mathbb{E}\left[e^{-\delta_{0}^{*} r_{b}} \mathbf{1}_{\left\{r_{b}<\infty\right\}} \mid \bar{U}_{b}^{\prime}(0)=0\right] \\
& =\mathbb{E}_{0}\left[e^{-\delta_{0}^{*} r_{b}} \mathbf{1}_{\left\{r_{b}<\infty\right\}}\right] \\
& =\frac{1}{Z^{\left(\delta_{0}^{*}\right)}(b)}
\end{aligned}
$$

and we complete the proof of Theorem 5 .

\section{Remarks:}

- Having calculated $W^{(p)}$ using the methods discussed in previous sections, $Z^{(p)}$ can be calculated accordingly.

- Through Laplace inversion (analytically or numerically) of $\bar{m}_{\delta^{*}}^{b}\left(u^{\prime}\right)$ and $\mathbb{E}_{u^{\prime}}\left[e^{-q D_{0}^{*}} \mathbf{1}_{\left\{\tau_{b}<\infty\right\}}\right]$ given in Theorem 4 and 5 , we can obtain the densities of $\tau_{b}$ and $D_{0}^{*}$. Then the densities of $T_{u}^{b}$ and $D_{0}$ can also be determined making use of the relationships between $\tau_{b}$ and $T_{u}^{b}$ as well as $D_{0}^{*}$ and $D_{0}$. 


\section{Numerical illustration}

In this section we present some numerical calculations on the Laplace transform of the non-dividend paying duration to illustrate how the distribution of this occupation time behaves in response to varying parameters and the impact of delay on the occupation times comparing to an ordinary Sparre Andersen risk model. Throughout this section we assume the inter-claim times $\left\{K_{i}\right\}_{i=2}^{\infty}$ follow a hyper-exponential distribution with p.d.f. $h(x)=\frac{1}{2} \alpha_{1} e^{-\alpha_{1} x}+\frac{1}{2} \alpha_{2} e^{-\alpha_{2} x}, x \geq 0$, then the Laplace exponent of $\left\{U^{\prime}(s): s \geq 0\right\}$ becomes

$$
\Psi(s)=c s-\lambda+\lambda\left(\frac{1}{2} \frac{\alpha_{1}}{\alpha_{1}+s}+\frac{1}{2} \frac{\alpha_{2}}{\alpha_{2}+s}\right), \quad \alpha_{1}<\alpha_{2},
$$

and explicit expressions for $W^{(\delta)}$ and $Z^{(\delta)}$ can be found in Example 1.1 of [30]. The set of parameters are: $\alpha_{1}=0.5, \alpha_{2}=1, \lambda=1.5$, and $c=1.2$. So $\mathbb{E}\left[K_{i}\right]=1.5, i=2,3, \ldots$, and the positive loading condition is satisfied with $\delta_{0}^{*}=0.05$. Considering a delayed Sparre Andersen risk model, i.e. $h_{0}(x)=e^{-x}$, Figure 7 shows $m_{b, 0}(u)$ for $u \in(0, b)$ given four different values of $b$. And Table 1 presents some selected values of $m_{b, 0}(u)$ with highlighted minimum values in each case.



Figure $7: m_{b, 0}(u)$ over $(0, b)$ given $b=1,3,5$ and 8

We know that the duration $D_{0}$ measures the total time that the surplus process spends within the interval $(0, b)$, with a lower barrier 0 and an upper barrier $b$, until the first exit from 0 . For a fixed $u$, it is intuitive that an increase in $b$ enlarges the interval and then decreases the chance that the surplus level exceeds $b$. Hence we should expect $D_{0}$ to increase, or equivalently, $m_{b, 0}(u)$ to decrease, which aligns with the trends shown in Figure 7 .

Another observation from Figure 7 is rather interesting. If we fix $b$ and allow $u$ to vary from 0 to $b$, the trend is different. An increase in $u$ has two effects on $D_{0}$ :

- firstly, it decreases the chance that the surplus level drops below 0 and hence has a positive impact on $D_{0}$; 
Table 1: $m_{b, 0}(u)$ for $u \in(0, b)$ given $b=1,3,5$ and 8

\begin{tabular}{cc|cc|cc|cc}
\hline$u$ & $m_{1,0}(u)$ & $u$ & $m_{3,0}(u)$ & $u$ & $m_{5,0}(u)$ & $u$ & $m_{8,0}(u)$ \\
\hline $\mathbf{0 . 0}$ & $\mathbf{0 . 9 4 9 7}$ & 0.0 & 0.8001 & 0.0 & 0.6611 & 0.0 & 0.5830 \\
0.1 & 0.9505 & 0.5 & 0.7489 & 1.0 & 0.4776 & 1.0 & 0.3426 \\
0.2 & 0.9518 & 1.0 & 0.7180 & 2.0 & 0.3857 & 2.0 & 0.2100 \\
0.3 & 0.9536 & 1.3 & 0.7072 & 2.5 & 0.3610 & 3.0 & 0.1379 \\
0.4 & 0.9559 & 1.6 & 0.7012 & 3.0 & 0.3461 & 4.0 & 0.0996 \\
0.5 & 0.9586 & $\mathbf{1 . 8 9 6 1}$ & $\mathbf{0 . 6 9 9 3}$ & 3.5 & 0.3387 & 5.0 & 0.0804 \\
0.6 & 0.9617 & 2.0 & 0.6996 & $\mathbf{3 . 8 9 6 1}$ & $\mathbf{0 . 3 3 7 0}$ & 6.0 & 0.0722 \\
0.7 & 0.9653 & 2.3 & 0.7025 & 4.0 & 0.3372 & $\mathbf{6 . 8 9 6 1}$ & $\mathbf{0 . 0 7 0 3}$ \\
0.8 & 0.9692 & 2.5 & 0.7061 & 4.5 & 0.3403 & 7.0 & 0.0704 \\
0.9 & 0.9735 & 2.8 & 0.7139 & 4.8 & 0.3441 & 7.5 & 0.0710 \\
\hline
\end{tabular}

- secondly, it increases the chance that the surplus level exceeds $b$ and hence has a negative impact on $D_{0}$.

The above two effects work together and produce the four trends shown in Figure 7.

- When $b$ is very small, i.e. $b=1$, an increase in $u$ results in a significant increase in the potential that the surplus will exceed $b$ but a relatively small protection against ruin. Therefore overall $D_{0}$ decreases.

- When $b$ is large, i.e. $b=8$, one can see a reverse trend which implies that an increase in $u$ significantly decreases the chance that the surplus will drop below 0 but has little impact on the potential of exceeding $b$. Therefore the overall trend for $D_{0}$ is increasing.

- For an intermediate $b$, when $u$ starts to increase from 0 , it provides a stronger protection against ruin rather than an assistance for the surplus to exceed $b$, hence $D_{0}$ increases initially. As $u$ keeps increasing, the former effect becomes less significant while the later one keeps enhancing and eventually becomes the dominating effect. This explains the convex type relationship between $u$ and $m_{b, 0}(u)$ when $b=3$ and $b=5$.

In order to access the impact of the premium rate $c$, we fix $b=3$ and consider four different values of $c$, i.e. $c=1.05,1.1,1.2,1.3$. Then we have the following Figure 8. Again, Table 2 presents some selected values of $m_{3,0}(u)$ with highlighted minimum values in each case. 


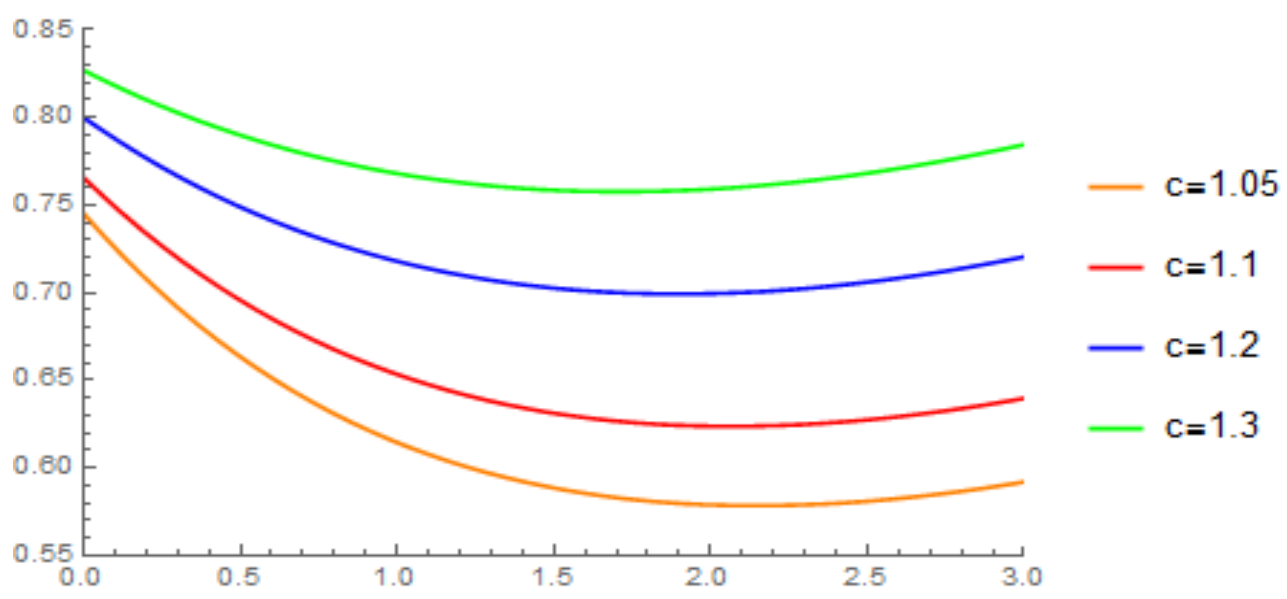

Figure 8: $m_{3,0}(u)$ over $(0,3)$ given $c=1.05,1.1,1.2$ and 1.3

Table 2: $m_{3,0}(u)$ for $u \in(0,3)$ given $c=1.05,1.1,1.2$ and 1.3

\begin{tabular}{cc|cc|cc|cc}
\hline$u$ & $c=1.05$ & $u$ & $c=1.1$ & $u$ & $c=1.2$ & $u$ & $c=1.3$ \\
\hline 0.0 & 0.7453 & 0.0 & 0.7658 & 0.0 & 0.8001 & 0.0 & 0.8271 \\
0.5 & 0.6634 & 0.5 & 0.6957 & 0.5 & 0.7489 & 0.5 & 0.7900 \\
1.0 & 0.6146 & 1.0 & 0.6534 & 1.0 & 0.7180 & 1.0 & 0.7681 \\
1.3 & 0.5967 & 1.3 & 0.6381 & 1.3 & 0.7072 & 1.3 & 0.7611 \\
1.6 & 0.5856 & 1.6 & 0.6288 & 1.6 & 0.7012 & 1.6 & 0.7580 \\
2.0 & 0.5790 & 2.0 & 0.6239 & $\mathbf{1 . 8 9 6 1}$ & $\mathbf{0 . 6 9 9 3}$ & $\mathbf{1 . 7 1 3 0}$ & $\mathbf{0 . 7 5 7 7}$ \\
$\mathbf{2 . 1 4 2 2}$ & $\mathbf{0 . 5 7 8 5}$ & $\mathbf{2 . 0 6 3 9}$ & $\mathbf{0 . 6 2 3 7}$ & 2.0 & 0.6996 & 2.0 & 0.7592 \\
2.3 & 0.5790 & 2.3 & 0.6249 & 2.3 & 0.7025 & 2.3 & 0.7637 \\
2.5 & 0.5811 & 2.5 & 0.6275 & 2.5 & 0.7061 & 2.5 & 0.7683 \\
2.8 & 0.5867 & 2.8 & 0.6340 & 2.8 & 0.7139 & 2.8 & 0.7773 \\
\hline
\end{tabular}

In Figure 8, for each $c$ level a convex relationship between $D_{0}$ and $u$ is evident which can be explained using a similar reasoning as above. Since the premium income enables the surplus process to drift to infinity, a larger $c$ increases the chance that the surplus exceeds $b$. Hence for a fixed $u$, as $c$ increases, $m_{b, 0}(u)$ increases and $D_{0}$ decreases. In addition, when $c$ decreases, the convex curve changes direction at a larger $u$, which implies that when the premium rate is lower, the initial surplus has a stronger impact on ruin protection. Hence when $u$ increases towards $b$, the effect of ruin protection dominates for a longer period and equivalently $D_{0}$ increases for a longer period.

Last but not least, we test the delay impact on $m_{b, 0}(u)$. We do this by fixing $b=3$ and making five different distributional assumptions on $h_{0}(x)$ that are all different from the one adopted before: for $x \geq 0$,

- case 1: $K_{1} \sim \operatorname{Pareto}(3,3), h_{0}(x)=\frac{3 \times 3^{3}}{(3+x)^{4}}$ 
- case $2: h_{0}=h$, i.e. $h_{0}(x)=\frac{1}{2} 0.5 e^{-0.5 x}+\frac{1}{2} e^{-x}$;

- case 3: $K_{1} \sim \operatorname{Exp}\left(\frac{2}{3}\right)$, i.e. $h_{0}(x)=\frac{2}{3} e^{-\frac{2}{3} x}$;

- case 4: $K_{1} \sim \operatorname{Gamma}(1.5,1), h_{0}(x)=e^{-x} \frac{x^{0.5}}{\Gamma(1.5)}$

- case 5: $K_{1} \sim \operatorname{Gamma}\left(2, \frac{4}{3}\right)$, i.e. $h_{0}(x)=\frac{16}{9} x e^{-\frac{4}{3} x}$.

We deliberately choose the densities such that they have equal means but decreasing variances.

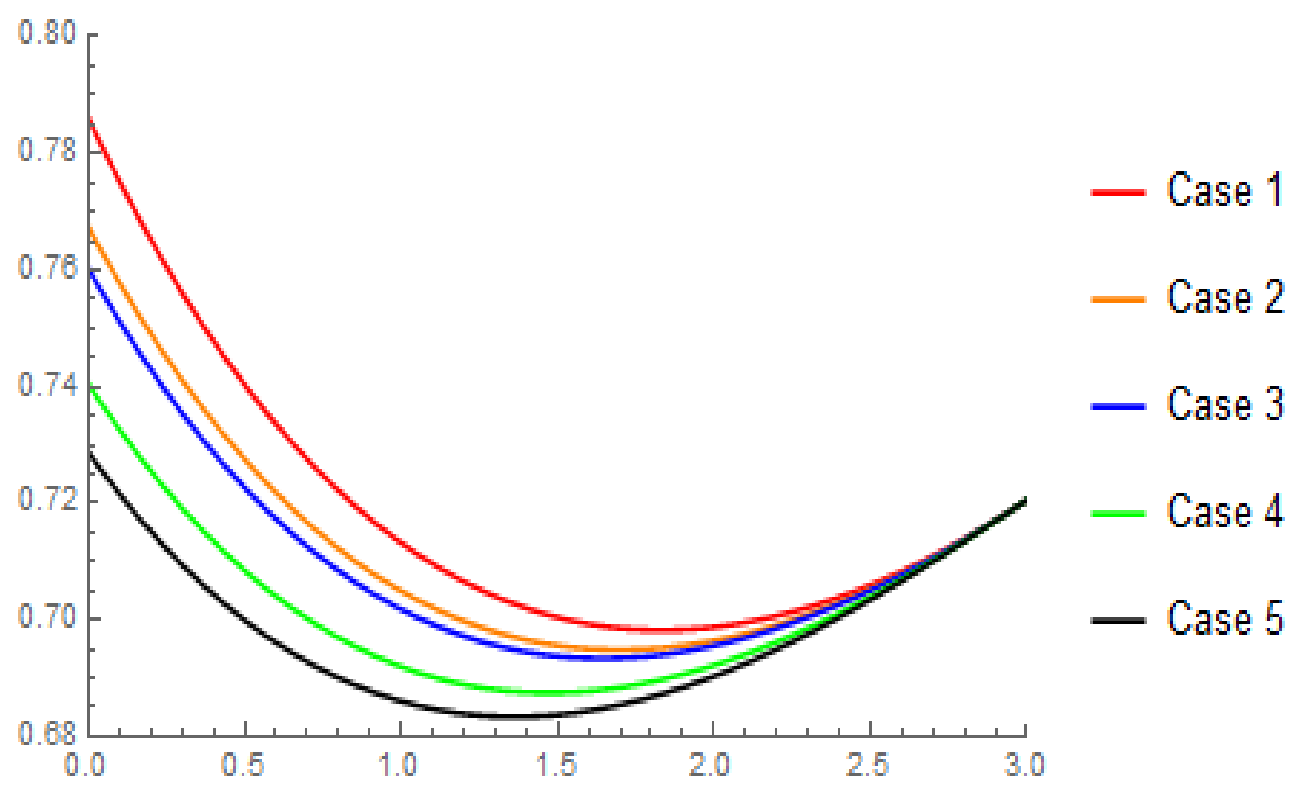

Figure 9: $m_{3,0}(u)$ over $(0,3)$ with various $h_{0}(x)$

The first observation is that different assumptions on $h_{0}$ do not change the general shape of the graph. More interestingly, we observe that $m_{b, 0}(u)$ increases with the coefficient of dispersion of $h_{0}$. For instance, the heavy-tailed Pareto density has the highest variance (so highest coefficient of dispersion) which gives a greater potential that the surplus process will receive a significant amount of premium before the first claim. This naturally results in an increased probability that the surplus will reach $b$ before the first claim and hence a potential decrease in $D_{0}$, or an increase in $m_{b, 0}(u)$ equivalently. Therefore we should expect the size of $m_{b, 0}(u)$ to be positively related to the coefficient of dispersion of $h_{0}$, which aligns with our observation.

Further, as the coefficient of dispersion of $h_{0}$ increases, the turning points of the convex curves move to the right, which suggests that a higher coefficient of dispersion increases the probability of collecting more premium before the first claim. Then the premium income before the first claim has a stronger impact than the initial surplus, which provides some degree of ruin protection. Hence $D_{0}$ increases over a wider range of $u$. Lastly, when $u$ is 
Table 3: $m_{3,0}(u)$ for $u \in(0,3)$ with various $h_{0}(x)$

\begin{tabular}{cc|cc|cc|cc|cc}
\hline$u$ & Case 1 & $u$ & Case 2 & $u$ & Case 3 & $u$ & Case 4 & $u$ & Case 5 \\
\hline 0.0 & 0.7861 & 0.0 & 0.7674 & 0.0 & 0.7603 & 0.0 & 0.7405 & 0.0 & 0.7287 \\
0.5 & 0.7403 & 0.5 & 0.7276 & 0.5 & 0.7153 & 0.5 & 0.7085 & 0.5 & 0.7000 \\
1.0 & 0.7132 & 1.0 & 0.7050 & 1.0 & 0.7018 & 1.0 & 0.6920 & 1.0 & 0.6861 \\
1.3 & 0.7041 & 1.3 & 0.6980 & 1.3 & 0.6957 & 1.3 & 0.6881 & 1.3 & 0.6839 \\
1.5 & 0.7004 & 1.5 & 0.6956 & 1.5 & 0.6938 & $\mathbf{1 . 4 7 1 4}$ & $\mathbf{0 . 6 8 7 5}$ & $\mathbf{1 . 3 6 2 2}$ & $\mathbf{0 . 6 8 3 4}$ \\
$\mathbf{1 . 9 0 3 2}$ & $\mathbf{0 . 6 9 8 4}$ & $\mathbf{1 . 6 9 7 7}$ & $\mathbf{0 . 6 9 4 9}$ & $\mathbf{1 . 6 4 1 8} \mathbf{0 . 6 9 3 4}$ & 1.6 & 0.6878 & 1.6 & 0.6845 \\
2.0 & 0.6988 & 2.0 & 0.6964 & 2.0 & 0.6956 & 2.0 & 0.6922 & 2.0 & 0.6904 \\
2.3 & 0.7022 & 2.3 & 0.7009 & 2.3 & 0.7005 & 2.3 & 0.6986 & 2.3 & 0.6976 \\
2.5 & 0.7060 & 2.5 & 0.7053 & 2.5 & 0.7051 & 2.5 & 0.7040 & 2.5 & 0.7035 \\
2.9 & 0.71713 & 2.9 & 0.71710 & 2.9 & 0.71709 & 2.9 & 0.71703 & 2.9 & 0.71701 \\
\hline
\end{tabular}

much less than $b$, a higher coefficient of dispersion of $h_{0}$ allows more room for the premium collection and hence we observe significant dispersion in $m_{b, 0}(u)$ (or $D_{0}$ ) when $u$ is small; however, when $u$ is close to $b$, a slightly longer time before the first claim would enables the surplus to exceed $b$. Therefore the impact of the variation in $h_{0}$ is limited and diminishes when $u$ approaches $b$, which results in the convergence at the right tail in the figure.

We remark that the numerical calculations and the corresponding analyses can be done in a similar way for the Laplace transforms obtained in Theorem 3 and 4 . And specific numerical methods for dealing with the corresponding inversion of Laplace transforms can be found in Section 5 of [17].

\section{Acknowledgment}

The authors would like to thank Prof. Xiaowen Zhou from Concordia University and an anonymous referee for their valuable comments and suggestions which resulted in significant improvements in both the form and the content of the paper.

\section{References}

[1] Albrecher, H., Claramunt, M. M., and Mármol, M. On the distribution of dividend payments in a Sparre Andersen model with generalized Erlang (n) interclaim times. Insurance: Mathematics and Economics 37, 2 (2005), 324-334.

[2] Albrecher, H., And Hartinger, J. A risk model with multilayer dividend strategy. North American Actuarial Journal 11, 2 (2007), 43-64.

[3] Albrecher, H., Hartinger, J., And Tichy, R. F. On the distribution of dividend payments and the discounted penalty function in a risk model with linear dividend barrier. Scandinavian Actuarial Journal 2005, 2 (2005), 103-126. 
[4] Albrecher, H., And Ivanovs, J. A risk model with an observer in a Markov environment. Risks 1, 3 (2013), 148-161.

[5] Albrecher, H., And Kainhofer, R. Risk theory with a nonlinear dividend barrier. Computing 68, 4 (2002), 289-311.

[6] Albrecher, H., Kainhofer, R., And Tichy, R. F. Simulation methods in ruin models with non-linear dividend barriers. Mathematics and Computers in Simulation 62, 3 (2003), 277-287.

[7] Badescu, A., Drekic, S., and Landriault, D. Analysis of a threshold dividend strategy for a MAP risk model. Scandinavian Actuarial Journal 2007, 4 (2007), 227247.

[8] Borovkov, K. A., And Dickson, D. C. On the ruin time distribution for a Sparre Andersen process with exponential claim sizes. Insurance: Mathematics and Economics 42, 3 (2008), 1104-1108.

[9] Breuer, L., ET Al. Occupation times for Markov-modulated Brownian motion. Journal of Applied Probability 49, 2 (2012), 549-565.

[10] De Finetti, B. Su unimpostazione alternativa della teoria collettiva del rischio. In Transactions of the XVth international congress of Actuaries (1957), vol. 2, pp. 433443.

[11] Dickson, D. C., And Egídio Dos Reis, A. D. On the distribution of the duration of negative surplus. Scandinavian Actuarial Journal 1996, 2 (1996), 148-164.

[12] Egídio Dos Reis, A. How long is the surplus below zero? Insurance: Mathematics and Economics 12, 1 (1993), 23-38.

[13] Gerber, H. U. On the probability of ruin in the presence of a linear dividend barrier. Scandinavian Actuarial Journal 1981, 2 (1981), 105-115.

[14] Gerber, H. U. When does the surplus reach a given target? Insurance: Mathematics and Economics 9, 2 (1990), 115-119.

[15] Gerber, H. U., And Shiu, E. S. The time value of ruin in a Sparre Andersen model. North American Actuarial Journal 9, 2 (2005), 49-69.

[16] Huang, Y., And Yu, W. The Gerber-Shiu discounted penalty function of Sparre Andersen risk model with a constant dividend barrier. Mathematical Problems in Engineering (2014).

[17] Kuznetsov, A., Kyprianou, A. E., and Rivero, V. The theory of scale functions for spectrally negative Lévy processes. In Lévy Matters II. Springer, 2013, pp. 97-186.

[18] Kyprianou, A. E. Introductory lectures on fluctuations of Lévy processes with applications. Springer Science \& Business Media, 2006. 
[19] Kyprianou, A. E. Gerber-Shiu risk theory. Springer Science \& Business Media, 2013.

[20] Landriault, D. Constant dividend barrier in a risk model with interclaim-dependent claim sizes. Insurance: Mathematics and Economics 42, 1 (2008), 31-38.

[21] Landriault, D., Renaud, J.-F., And Zhou, X. Occupation times of spectrally negative Lévy processes with applications. Stochastic processes and their applications 121, 11 (2011), 2629-2641.

[22] Landriault, D., AND Shi, T. Occupation times in the MAP risk model. Insurance: Mathematics and Economics 60 (2015), 75-82.

[23] Li, B., Zhou, X., et AL. The joint Laplace transforms for diffusion occupation times. Advances in Applied Probability 45, 4 (2013), 1049-1067.

[24] Li, S., ANd Garrido, J. On a class of renewal risk models with a constant dividend barrier. Insurance: Mathematics and Economics 35, 3 (2004), 691-701.

[25] Li, S., And Garrido, J. On ruin for the Erlang (n) risk process. Insurance: Mathematics and Economics 34, 3 (2004), 391-408.

[26] Li, S., AND Lu, Y. The distribution of total dividend payments in a Sparre Andersen model. Statistics and Probability Letters 79, 9 (2009), 1246-1251.

[27] Li, Y., AND Zhou, X. On pre-exit joint occupation times for spectrally negative Lévy processes. Statistics and Probability Letters 94 (2014), 48-55.

[28] Lin, X. S., And Pavlova, K. P. The compound Poisson risk model with a threshold dividend strategy. Insurance: Mathematics and Economics 38, 1 (2006), 57-80.

[29] Lin, X. S., Willmot, G. E., And Drekic, S. The classical risk model with a constant dividend barrier: analysis of the Gerber-Shiu discounted penalty function. Insurance: Mathematics and Economics 33, 3 (2003), 551-566.

[30] Loeffen, R. L., Renaud, J.-F., And Zhou, X. Occupation times of intervals until first passage times for spectrally negative Lévy processes. Stochastic Processes and their Applications 124, 3 (2014), 1408-1435.

[31] Ramaswami, V. Passage times in fluid models with application to risk processes. Methodology and Computing in Applied Probability 8, 4 (2006), 497-515.

[32] Shi, T., And Landriault, D. Distribution of the time to ruin in some Sparre Andersen risk models. ASTIN Bulletin 43, 01 (2013), 39-59.

[33] Yuen, K. C., Wang, G., And Li, W. K. The Gerber-Shiu expected discounted penalty function for risk processes with interest and a constant dividend barrier. Insurance: Mathematics and Economics 40, 1 (2007), 104-112. 
[34] ZhaO, C., AND Zhang, C. Joint density of the number of claims until ruin and the time to ruin in the delayed renewal risk model with Erlang (n) claims. Journal of Computational and Applied Mathematics 244 (2013), 102-114.

[35] Zhou, X. On a classical risk model with a constant dividend barrier. North American Actuarial Journal 9, 4 (2005), 95-108. 


\section{University Library}

\section{- M M N E R VA A gateway to Melbourne's research publications}

Minerva Access is the Institutional Repository of The University of Melbourne

Author/s:

Jin, C;Li, S;Wu, X

Title:

On the occupation times in a delayed Sparre Andersen risk model with exponential claims

Date:

2016-11-01

Citation:

Jin, C., Li, S. \& Wu, X. (2016). On the occupation times in a delayed Sparre Andersen risk model with exponential claims. INSURANCE MATHEMATICS \& ECONOMICS, 71, pp.304-316. https://doi.org/10.1016/j.insmatheco.2016.10.001.

Persistent Link:

http://hdl.handle.net/11343/118606 